\title{
Accurate, ultra-low coverage genome reconstruction and association studies in 2 Hybrid Swarm mapping populations
}

4 Cory A. Weller ${ }^{1,2^{*}}$, Susanne Tilk ${ }^{3}$, Subhash Rajpurohit ${ }^{4}$, Alan O. Bergland ${ }^{1}$

$6{ }^{1}$ Department of Biology, University of Virginia, Charlottesville, Virginia 22904

$8{ }^{2}$ National Human Genome Research Institute, National Institutes of Health, Bethesda, MD 20892

10 3Department of Biology, Stanford University, Stanford CA 94305

${ }^{4}$ Department of Biological and Life Sciences, Ahmedabad University, Ahmedabad, India

12380009

14

* To whom correspondence should be addressed: cory.weller@nih.gov 
20 Genetic association studies seek to uncover the link between genotype and phenotype, and often utilize inbred reference panels as a replicable source of genetic variation.

22 However, inbred reference panels can differ substantially from wild populations in their genotypic distribution, patterns of linkage-disequilibrium, and nucleotide diversity. As a

24 result, associations discovered using inbred reference panels may not reflect the genetic basis of phenotypic variation in natural populations. To address this problem,

26 we evaluated a mapping population design where dozens to hundreds of inbred lines are outbred for few generations, which we call the Hybrid Swarm. The Hybrid Swarm

28 approach has likely remained underutilized relative to pre-sequenced inbred lines due to the costs of genome-wide genotyping. To reduce sequencing costs and make the

30 Hybrid Swarm approach feasible, we developed a computational pipeline that reconstructs accurate whole genomes from ultra-low-coverage $(0.05 \mathrm{X})$ sequence data

32 in Hybrid Swarm populations derived from ancestors with phased haplotypes. We evaluate reconstructions using genetic variation from the Drosophila Genetic Reference

34 Panel as well as variation from neutral simulations. We compared the power and precision of GWAS using the Hybrid Swarm, inbred lines, recombinant inbred lines, and

36 highly outbred populations across a range of allele frequencies, effect sizes, and genetic architectures. Our simulations show that these different mapping panels vary in

38 their power and precision, largely depending on the architecture of the trait. The Hybrid Swam and RILs outperform inbred lines for quantitative traits, but not for monogenic

40 ones. Taken together, our results demonstrate the feasibility of the Hybrid Swarm as a cost-effective method of fine-scale genetic mapping. 


\section{Introduction}

44 Genetic mapping studies seek to describe the link between genotype and phenotype. For experimental crosses, mapping was traditionally conducted by scoring the

46 phenotypes of recombinant offspring descended from a limited number of parental lines (Lander and Botstein 1989). While such QTL mapping studies can have high power to

48 detect association, they offer minimal mapping resolution (Cheng et al. 2010), often detecting broad regions of phenotypic association. If linkage disequilibrium is lowered,

50 regions of association can be resolved at the gene or nucleotide level ( $\mathrm{Li}$ et al. 2005; Rockman and Kruglyak 2008), as in GWAS of large outbred populations (Nikpay et al.

52 2015; Wu et al. 2017; Monir and Zhu 2017). However, GWAS suffer from reduced power to detect associations (Long and Langley 1999), necessitating a large sample

54 size relative to QTL mapping (Spencer et al. 2009).

56 To generate higher resolution mapping populations than the traditional biparental crosses, Multiparental Populations (MPPs) are becoming increasingly used. By crossing

58 together multiple inbred lines, one can produce genetically diverse mapping populations without sampling wild individuals. MPPs are commonly used for the dissection of

60 complex traits in model organisms (Chesler et al. 2008; Kover et al. 2009; King et al. 2012) and agriculturally important crops (Huang et al. 2012a; Singh et al. 2013; Krämer

62 et al. 2014). Although MPPs are statistically powerful (Valdar et al. 2006; Svenson et al. 2012), they may have limited utility in addressing basic questions about the evolutionary

64 forces affecting causal variants in natural populations (Long et al. 2014) and are often restricted to existing mapping panels in a limited number of taxa.

The mapping resolution of MPPs depends on the extent of linkage disequilibrium.

68 Mapping resolution is improved by allowing for more recombination between haplotypes or by substituting extensive recombination for increased haplotype diversity (Mott et al.

70 2000; Chia et al. 2005). In the latter approach, by crossing dozens to hundreds of inbred lines for a limited number $(\sim 5)$ of generations and subsequently phenotyping and

72 genotyping outbred individuals, heterozygous mapping populations can be generated quickly with sufficiently reduced LD to potentially detect associations with high

74 resolution. We refer to such an outbred mapping population as a Hybrid Swarm. Whether Hybrid Swarm mapping populations can be generated efficiently, and whether

76 association mapping using such a population is useful, remain open questions.

78 To address these questions, we evaluate a method to reconstruct phased whole genomes from large Hybrid Swarm populations using ultra-shallow sequencing $(<0.05 X)$

80 and assessed the power and precision of association mapping using a Hybrid Swarm relative to common alternatives [8-way MPP - DSPR (Long et al. 2014); inbred lines -

82 DGRP (MacKay et al. 2012)]. First, we developed and tested our genome reconstruction method by generating whole genomes for thousands of simulated Hybrid

84 Swarm individuals. Our simulated genomes draw from natural variation in the Drosophila melanogaster Genetic Reference Panel (DGRP), or variation generated from

86 coalescent models representing a broad range of genetic diversity parameters for common model systems. We show that the Hybrid Swarm approach allows for highly accurate genotyping (average $99.9 \%$ genotypic accuracy) from ultra-low-coverage 
whole-genome individual-based sequencing. We confirm the accuracy of genome

90 reconstructions using experimental animals from a Hybrid Swarm constructed in the lab from 128 DGRP founder lines. We then simulated a range of genetic architectures to

92 discern the accuracy and precision of association mapping in the Hybrid Swarm compared to inbred lines, recombinant inbred lines, and a highly outbred population.

94 GWA simulations confirm that inbred MPPs have the highest power to detect associations, outbred Hybrid Swarm populations have intermediate power, and inbred

96 reference panels have the lowest power, particularly for quantitative traits. In addition, we show that outbred Hybrid Swarms eliminate spurious signals of association that

98 arise using inbred lines. Together, our results show the feasibility of cost-effective association mapping in a large outbred multi-parental population and provides tools for

100 genome-reconstruction and simulation.

\section{Methods}

Simulating a Hybrid Swarm. As a case study of low-coverage genome reconstruction

104 in a model system, we simulated a Hybrid Swarm using sequenced inbred lines from the Drosophila Genetic Reference Panel (MacKay et al. 2012) as available from the

106 Drosophila Genome Nexus (Lack et al. 2015). We only included the 128 lines (out of 205) with the least amount of missing genotype data. We removed insertions, deletions,

108 and sites with more than two alleles. Any heterozygous genotype calls were masked as missing data.

To generate simulated populations, we developed a forward-simulator in $\mathrm{R}$ that stores

112 ancestral haplotype block maps instead of genotypes. See Figure 1 for depictions of mapping populations simulated. We simulated Hybrid Swarms through random mating

114 over five non-overlapping generations at a population size of 10,000. Sexual reproduction was simulated by random sampling of recombinant gametes from male-

116 female pairs. Recombination frequency was modeled as a Poisson process with an expected value $\lambda=\Sigma$ (Morgans) per chromosome. For simulations of Drosophila

118 populations based on DGRP chromosomes, recombination occurred only in females, with recombination frequency and position based on values from Comeron et al (2012).

120 See extended methods and Supplemental Figure S1 for additional detail.

122 We also simulated Hybrid Swarms using haplotypes derived from whole-genome coalescent simulation using scrm (Staab et al. 2015). We generated simulations across

124 a range of diversity and recombination levels, encompassing the values for many common model systems. For populations founded by simulated haplotypes,

126 recombination occurred in both sexes, with recombination occurring uniformly across each chromosome.

We used $w g \operatorname{sim}$ ( $\mathrm{Li} 2011$ ) to simulate $100 \mathrm{bp}$ reads at two average read depths $(0.005 \mathrm{X}$ and $0.05 \mathrm{X}$ ). We specified a base error rate of 0.001 and an indel fraction of 0 . Remaining wgsim parameters were left as default. We assembled paired end reads

132 using PEAR (Zhang et al. 2014) and separately aligned the assembled and unassembled groups to a reference genome with bwa 0.7.14 using the BWA-MEM 
134 algorithm (Li 2013). Simulated Drosophila reads were mapped to the D. melanogaster reference genome v5.39. After converting mapped reads to compressed BAM format

136 with samtools 1.3.1 (Li et al. 2009), we removed PCR duplicates with Picard tools 2.0.1 ("Picard toolkit" 2019).

Genome reconstruction for a Hybrid Swarm. Genome reconstruction for Hybrid

140 Swarm individuals can take advantage of Hidden Markov Models developed for standard MPPs (Zheng et al. 2015b). In these models, the probability of each diplotype

142 - a unique diploid combination of founding haplotypes - is calculated. Calculating these probabilities is feasible with smaller numbers of founding lines ( $<8$, typically) but grows

144 at a quadratic rate. Therefore, increasing the number of founding haplotypes from 8 to 128 incurs orders of magnitude more computational effort (Supplemental Figure S2)

146 and is not feasible. To make chromosome reconstructions in the Hybrid Swarm computationally tractable, we developed a method of accurately selecting a subset of

148 most likely ancestors for any single chromosome. It is important to note that the computational requirement of selecting a subset of founding chromosomes requires that

150 a Hybrid Swarm only be propagated for a limited number of generations, especially if the number of founding lines is large.

We used the software package HARP (Kessner et al. 2013) to identify the most likely

154 founding lines based on the number of genomic windows with high probabilities of ancestry. HARP was originally developed to estimate haplotype frequencies from

156 pooled sequence data, and we co-opted it to assess relative likelihood that any founder contributed to a genomic window in a focal Hybrid Swarm chromosome. We ran HARP

158 with non-overlapping $100 \mathrm{~kb}$ windows with a minimum frequency cutoff 0.0001 . Ideally, any given window would have four (or fewer) founders with high proportions of ancestry

160 ( 25\%) and unambiguous haplotype paths (Supplemental Figure S3). However, empirical evaluation of Harp output for sample chromosomes showed that many had

162 more than four founders with high proportion of ancestry and difficult-to-resolve haplotypes (Supplemental Figure S4).

We developed a heuristic method to identify the set of most likely ancestors. We ranked

166 all possible founders by their contribution to the focal chromosome. To do this, we counted the number of windows with extreme likelihoods of ancestry (i.e., greater than a

168 specified quantile) for each potential founding haplotype. Potential founding lines were then ranked by the number of windows passing this threshold. We examined two

170 measures of effectiveness for this method across a range of quantile threshold values (90\%, 95\%, 99\%, and 99.9\%) when selecting up to a maximum number of most likely

172 ancestral founders. The first measure is the number of true ancestral founders excluded; the second measure is the fraction of the chromosome derived from

174 ancestors missing from the selected subset.

176 We used the Mathematica package RABBIT (Zheng et al. 2015a) to perform chromosome reconstructions. This package has been shown to be accurate for

178 genotype estimation at sequencing coverage at $0.05 \mathrm{X}$ for a variety of multiparent populations (Zheng et al. 2018) but, the best of our knowledge, only for MPPs with 
180 twelve or fewer potential founders. To input the observed genotype of each recombinant individual, we counted reference and alternate reads at variable sites (i.e. polymorphic

182 among the list of potential founders) using the Genome Analysis Toolkit ASEReadCounter tool (Broad Institute 2015). Because it is not possible to make

184 confident homozygote genotype calls from low coverage sequencing data where most sites are observed only once and or twice, we encoded individuals with only reference

186 or alternate alleles as "1N" or "2N"; observed heterozygotes were encoded as "12". We sampled 5000 highly informative SNPs per individual per chromosome for genome

188 reconstruction. Informative SNPs were identified as those at low frequency amongst potential founders identified in the HARP step. We ran RABBIT independently for each

190 chromosome using the Viterbi decoding function under the "joint model" with all other RABBIT parameters left at default. RABBIT output was converted to a phased

192 chromosome haplotype map, which we then used to extract and concatenate genotype information from a VCF file containing founder genotypes.

We evaluated the accuracy of reconstruction by calculating genotype accuracy and the number of inferred recombination events. To calculate genotype accuracy, we measured the fraction of sites where the estimated diploid genotype is identical to the

198 originally simulated diploid genotype. We only examined accuracy on the autosomes. To measure accuracy of estimated frequency of recombination events, true and

200 estimated recombination counts were first summed over both copies of each chromosome in a simulated individual. We then calculated Lin's concordance

202 correlation coefficient $\rho$ between the true and estimated recombination counts using the epi.ccc function of the $R$ package epiR (Stevenson 2018). An overview of our

204 reconstruction pipeline is shown in Supplemental Figure S5.

206 Chromosome reconstruction with STITCH. We estimated genotypes with alternative approach, STITCH, which is capable of imputing genotypes without reference panels

208 (Davies et al. 2016). To assess genotype estimation accuracy using STITCH, we generated and mapped simulated reads for chromosome $2 \mathrm{~L}$ at $0.05 \mathrm{X}$ coverage for 5000

210 individuals in a $F_{5}$ Hybrid Swarm population ( $N=10000$ individuals per generation). Because STITCH draws inference from haplotypes between related individuals,

212 genotype estimate accuracy is expected to increase with greater numbers of sequenced individuals. To capture the effect of sample size on STITCH accuracy, we ran STITCH

214 for sample sizes from 100 to 5000 sequenced individuals. We calculated genotype estimate accuracy as the fraction of variable sites with a correct diploid genotype

216 estimate using a custom R script. Because STITCH memory requirements increase with greater values of $k$ (founding haplotypes), it was not possible to evaluate $k=128$

218 founders, or to use the diploid estimation model. As a result, we limited our evaluations to 32-founder populations, using the pseudoHaploid model.

Generating a real Hybrid Swarm population. We generated a hybrid swarm mapping 222 population through undirected mating of all Drosophila Genetic Reference Panel (DGRP) inbred lines (Mackay et al. 2012) in large population cages (6' x 6' x 6').

224 Populations were seeded with males and females from each line and were expanded for four generations on cornmeal-molasses media. Cages reached an approximate final 
226 population size of $\sim 500,000$ as determined by a volumetric assay of dead adults. Generations were discrete: we removed egg-laden media from the cages and replaced egg-laden media in emptied cages after removal of the previous generation.

230 Library preparation and read mapping. We homogenized individual flies in $350 \mu \mathrm{L}$ Lysis Buffer RLT Plus using 2-3 $1 \mathrm{~mm}$ beads in a bead shaker for 2 minutes. DNA and

232 RNA were extracted using the AllPrep DNA/RNA Micro Kit (Qiagen product number 80284). Following DNA extraction, we purified samples with Ampure XP Beads

234 (Beckman Coulter product number A63880). We prepared $1 \mu \mathrm{L}$ of DNA at $\sim 2.5 \mathrm{ng} / \mu \mathrm{L}$ sequencing using a modified Nextera protocol developed by Baym et al. (2015),

236 indexing samples with custom primers, and selecting fragments between 450-500 bp in length using a SizeSelect e-Gel. As a final step to amplify the prepared DNA

238 sequencing libraries, we ran all size selected samples through additional 5 rounds of PCR. Each PCR reaction used $5 \mu \mathrm{L}$ template DNA, $0.6 \mu \mathrm{L}$ of $100 \mathrm{mM}$ forward and

240 reverse primers (custom synthesized by IDT), 10 $\mu \mathrm{L}$ of KAPA HiFi Ready Mix (KAPA Biosystem product number KK2611/2612), and $3.8 \mu \mathrm{L}$ nuclease free water. Our PCR

242 protocol included 5 minutes of initial denaturation at $95^{\circ} \mathrm{C}$ followed by 4 rounds of 20 seconds denaturation $\left(98^{\circ} \mathrm{C}\right), 20$ seconds annealing $\left(62^{\circ} \mathrm{C}\right), 30$ seconds elongation

$244\left(72^{\circ} \mathrm{C}\right)$, followed by a final elongation at $72^{\circ} \mathrm{C}$ for 2 minutes. Following PCR amplification, we purified DNA libraries using Ampure XP beads and quantified

246 concentrations on a Life Technologies Qubit spectrophotometer and Agilent Bioanalyzer. We sequenced the same library preparations at low ( 0.05X) and high ( 8)

248 coverage on separate Illumina sequencing runs. Reads were mapped to the reference genome using the same methods as our simulations.

Empirical evaluation of genotype accuracy in the Hybrid Swarm. We tested if

252 RABBIT genotype estimates accurately reflected genotypes from higher coverage data for six individuals. First, we called genotypes in the high coverage samples using a

254 heuristic based on reference and alternate allele counts produced by GATK's ASEReadCounter to call genotypes. Sites with at least three reference and three 256 alternate allele bearing short-reads were called as heterozygous; any site with at least six reference reads and zero alternate reads (or the converse) was called as

258 homozygous. All other sites with fewer six reads were excluded. We calculated accuracy as the proportion of sites where the low-coverage genotype derived from

260 reconstruction matched our higher-coverage genotype call. We note that our measurement of genotype accuracy from the empirical data is conservative as we 262 expect a moderate fraction of true heterozygous sites to be called as homozygous.

264 Simulating mapping populations for GWAS. We performed GWAS on simulations of various types of Hybrid Swarm designs and contrasted the signal of association using 266 these designs to that from a simulated 8-way MPP (akin to the DSPR), and the DGRP. Simulation of GWAS proceeded in two steps.

For every type of genetic architecture or mapping population explored, we simulated

270500 mapping populations comprised of 5000 individuals. Full genomes for individuals were generated using the forward simulation framework described above. We used the 
272 DGRP as the founder chromosomes. One important note is that the genotypes we use for GWAS are the "true" genotypes and are not affected by genotyping error. This

274 decision was made to reduce computation time. Because genotyping error is randomly distributed throughout the genome (and rare on a per-site basis, see Figure 2), the use

276 of perfect genotype data should not bias the results of our simulations.

278 Next, we assigned phenotypes to each simulated individual. We assign case or control phenotypes to individuals based on a liability model, where an observable binary

280 phenotype is produced from an underlying continuous trait, known as its liability (Xu and Atchley 1996). Under this model, phenotypic variance will increase with additional

282 causal loci. We contrasted mapping results for a monogenic trait and a polygenic trait, using loci with large and small effects, assigning individuals to case or control groups

284 based on their genotype at causal loci. We randomly selected a causal locus (or multiple loci for the polygenic model) from all variable sites in the mapping population.

286 We assigned the effect size of this locus to be proportional to its frequency: low frequency variants had a large effect and common variants had a small effect

288 (Supplemental Figure S6). To achieve a population size of 5000, it is necessary to sample from the 128 inbred lines or 800 recombinant inbred lines with replacement.

To test for association between phenotype and each SNP, we performed a $X^{2}$ test of 292 independence for reference and alternate allele counts between case and control groups. For inbred mapping populations, we corrected for non-independent allele draws 294 by dividing the $\mathrm{X}^{2}$ value by two.

296 Assessing GWAS accuracy. To measure GWAS accuracy, we generated Receiver Operator Characteristic (ROC) curves and calculated Area Under the Curve (AUC).

298 Briefly, we calculated the true positive rate (sensitivity) as a function of false positive rate (1-specificty) using the $R$ package $p R O C$ (Turck et al. 2011). In our simulations,

300 only the causal SNP may be considered a true positive. We summarize GWAS accuracy by averaging ROC curves from all replicate simulations, generating a single

302 representative ROC curve following Marigorta et al. (2018). We tested for statistical differences in the ROC curves between different mapping designs using a Wilcoxon test

304 on the distribution of simulated AUC values. We performed multiple-testing correction for the tests comparing AUC distributions using the Bonferroni method.

Genomic inflation factor. We calculated the genomic inflation factor (GIF) to evaluate the role of mapping design and genetic architecture on the overall signal of association. We evaluated GIF on chromosomes linked and unlinked to causal loci. We calculated

310 the GIF by correcting for sample size following Freedman et al (2004).

312 Data availability statement. The raw sequence data for our six reconstructed real-life hybrid swarm individuals is available at SRA BioProject accession PRJNA691163. The

314 most up-to-date code associated with this project is available on GitHub: https://github.com/cory-weller/HS-reconstruction-gwas and an archived version of our

316 repository is available at Zenodo (DOI: 10.5281/zenodo.4472955). This repository includes a containerized version of the reconstruction pipeline along with a test data-set. 


\section{Results}

Genome reconstruction for a Hybrid Swarm. First, we evaluated our most-likely ancestor selection algorithm, which selects a minimum representative set of MostLikely-Ancestors (MLAs). This step required optimizing a discrimination threshold for

324 likelihood values calculated with HARP (Kessner et al. 2013, see methods). We found that classifying any potential founder as an MLA by counting the number of

326 chromosomal windows with observed likelihoods in the top 5\% (threshold of 0.95 ) identified all potential founders with high accuracy (Supplemental Figure S7). The threshold of 0.99 performed slightly better when 128 founding lines were used to found a population, while the threshold of 0.95 performed slightly better with only 32 founding

330 lines. HARP thresholds of 0.95 and 0.99 perform similarly well across a range of simulated population parameters, suggesting this is a reasonable starting point for optimizing the pipeline for other populations (Supplemental Figure S8A). Reducing sequencing coverage by a factor of ten (from $0.05 \mathrm{X}$ to $0.005 \mathrm{X}$ ) resulted in similar levels

334 of ancestor selection accuracy (Supplemental Figure S8B), demonstrating that additional coverage may not necessarily improve reconstruction accuracy. For

336 simplicity, we next conducted all genome reconstructions with the 0.99 threshold at a simulated sequencing coverage of $0.05 \mathrm{X}$.

Next, we evaluated the accuracy of genotype calls following genome reconstruction

340 based on the most-likely ancestors identified for any individual chromosome. In general, genotype reconstruction from simulated $\mathrm{F}_{5}$ Hybrid Swarm individuals was very accurate: the median percent of sites with correctly estimated genotypes was greater than $99.9 \%$ whether the population was founded by 32 or 128 founding lines or made use of DGRP

344 or simulated haplotypes (Figure 2, Supplemental Figure S10). For simulations founded by DGRP lines, $80.5 \%$ of reconstructed chromosomes from 32 -founder populations

346 exhibited $>99.9 \%$ accuracy, with the remaining $19.5 \%$ of reconstructions contributing to a long tail with a minimum of $84.5 \%$. Increasing the number of founding lines to 128

348 resulted in genotype accuracy above $99 \%$ for all cases (minimum: $99.4 \%$ ), with $83 \%$ of reconstructed chromosomes achieving greater than $99.9 \%$ accuracy. Reducing

350 sequencing coverage by an order of magnitude from $0.05 \mathrm{X}$ to $0.005 \mathrm{X}$ resulted in more frequent over-estimation of recombination, though overall median genotype accuracy remained above 99\% (Supplemental Figure S9). Using simulated haplotypes, we also show that reconstruction accuracy improves with genetic diversity (Supplemental Figure 354 S10).

356 We also examined the accuracy of our estimates of recombination number, per individual. The number of recombination events estimated from was generally high but

358 differed between Hybrid Swarm populations with different numbers of founders. Reconstructions of individuals from Hybrid Swarm populations with more founders

360 generated more accurate estimates of recombination count (Lin's concordance correlation coefficient: $98 \%$ and $50 \%$ for DGRP 128- and 32-way Hybrid Swarms,

362 respectively; see Table 1). For 32-founder populations, DGRP-derived reconstructions 
tended to over-estimate recombination counts. Results on recombination count accuracy from simulated haplotypes are reported in Supplemental Table 1.

366 Although genome reconstruction using RABBIT accurately calls genotypes, and generally estimates the number of recombination events well, we observe some

368 reconstructions that are hyper-recombinant (i.e. $>10$ recombination events for our simulations of an F5, Figures 2 and 3). These hyper-recombinant individuals had among the lowest genotyping accuracy, and this was the case for both DGRP-derived and coalescent-derived hybrid swarm individuals. The cause of these hyper-recombinant 372 reconstructions is not always clear, but frequently appears due to RABBIT's estimation switching between closely-related haplotypes. Because the number of inferred recombination events is determined by the number of generations of recombination, hyper-recombinant individuals (or regions of a genome) can be easily identified and 376 removed as part of quality control (see Erickson et al 2020).

378 We contrasted the accuracy of genotype calls made with RABBIT to those made with an alternative tool, STITCH (Davies et al. 2016). STITCH does not rely on priors of parental haplotypes, but instead performs imputation and phasing based on patterns of linkage among the entire set of sequenced Hybrid Swarm recombinants. As expected, STITCH

382 accuracy improved with greater sample size of $N$ sequenced individuals (Figure S11), providing $68.5 \%$ genotype accuracy with $N=100$, increasingly approximately linearly until $94.5 \%$ accuracy at $N=3000$, and providing $99 \%$ accuracy with $N=4000$.

386 Genome reconstruction and genotype calls of real Hybrid Swarm individuals sequenced at ultra-shallow $(\sim 0.05 X)$ and high $(\sim 8)$ coverage were highly concordant $(\sim 95 \%$; Figure $3883)$. Accuracy is particularly high for reconstructed chromosomes that predict fewer than 10 recombination events.

392 Swarms and compared these to simulations of GWAS using an 8-way RILs (modeled after the DSPR), and an inbred reference panel (the DGRP). Examples of

394 representative Manhattan plots for a single causal locus of large effect are shown in Figure 4. RILs tend to result in a strong and wide association peak near the causal 396 allele, while other populations result in only a small number of sites (typically on or neighboring the causal locus) with $p$-values below $10^{-5}$.

To assess overall performance of these mapping populations across hundreds of

400 simulations, we generated Receiver Operator Characteristic (ROC) curves and calculated Area Under the Curve (AUC; Figure 5). In general, recombinant inbred lines

402 outperformed other mapping designed. RILs were only significantly outperformed for a single-locus trait of large effect mapped in a 128-founder inbred reference panel, though 404 both populations approach a median AUC of 1.0 (Figure 5B, median RIL AUC $=0.997$, median 32-founder Inbred Line AUC $\left.=0.999, p=1.101 \times 10^{-6}\right)$. Inbred reference panels 406 consistently had the lowest AUC for multi-locus traits (Figure 5C-F). We suspect that the decreased accuracy of association mapping using inbred lines is due to linkage to 408 causal sites, as well as spurious long-distance linkage (see below, Genomic Inflation 
410 types.

Factor). See Supplemental Table S2 for comparisons between all mapping population

412 Hybrid Swarm populations, composed of either 32- or 128-founders intermated for 5 generations had intermediate performance (Figure 5), and generally resembled fully

414 outbred (F50) populations composed of 128 founders. We also evaluated the GWAS performance when crossing 32 or 128 founders to generate F1 and F2 mapping

416 populations (Supplemental Figure S12). For single-locus simulations, the number of generations of recombination did not influence accuracy, although 32-founder

418 populations performed better than 128-founders. For multi-locus simulations, GWAS accuracy improved with more generations of recombination, and this effect was more

420 pronounced with 10-locus traits compared to 5-locus traits. Statistical tests comparing different models can be found in Supplemental Table S2.

Genomic Inflation Factor. An alternative way to assess the quality of GWAS using

424 different mapping designs is to compare the Genomic Inflation Factor. This metric describes how much the genome-wide distribution of test statistics differs from a null

426 expectation. Values greater than 1 can indicate an excess of low p-values and can reflect population structure (Reich and Goldstein 2000) or polygenicity (Yang et al.

428 2011). We calculated the genomic inflation factor for any given mapping population and genetic architecture genome-wide (across all autosomes), on the autosome arm

430 containing the causal allele (linked), and for sites on the autosome physically unlinked to the causal allele.

434 architecture with Inbred Lines producing the greatest GIF, and outbred F50 populations producing the lowest GIF (Figure 6). Hybrid Swarm populations showed intermediate

436 GIF. Interestingly, Inbred Lines display elevated GIF even for sites physically unlinked to causal loci, suggesting spurious long-distance linkage disequilibrium. This inflation on 438 unlinked chromosomes for inbred lines persists when filtering out sites below 5\% frequency (Supplemental Figure S13), suggesting the pattern cannot be attributed to

440 low-frequency alleles alone. Elevated GIF on unlinked chromosomes when using inbred panels such as the DGRP suggests caution in interpreting GWAS using this type of

442 mapping population, and could contribute to the observed differences in mapping results of the same trait studied in both inbred lines and an advanced Hybrid Swarms

444 derived from the DGRP (Huang et al. 2012b, 2020). The Hybrid Swarm, propagated for at least two generations, was able to decrease GIF at unlinked loci and by five

446 generations the problem of elevated GIF on unliked chromosomes was ameliorated.

\section{Discussion}

Here, we show that the Hybrid Swarm is a viable method for association mapping using

450 experimental outbred populations. We first demonstrate that ultra-low coverage, individual-based whole genome sequencing can be used to reconstruct accurate diploid 452 genomes for species across a range of diversity levels (Figure 2, Supplemental Figure S10), and we validate the quality of genome reconstruction using real Hybrid Swarm

454 individuals sequenced at both high and low coverages (Figure 3). Genome 
reconstruction with a reduced subset of most-likely-ancestors is computationally efficient (Supplemental Figure S2), and not dependent upon the number of individuals genotyped unlike STITCH (Supplemental Figure S11), enabling a wide range of

458 experimental designs. Next, we demonstrate that association mapping using the Hybrid Swarm can match or outperform various types of inbred lines and completely outbred

460 populations (Figures 4, 5, Supplemental Figure S12, Supplemental Table S2). Lastly, we have described a set of computational tools to simulate various mapping populations (including genome reconstructions, and GWAS), enabling this method to easily be applied to a variety of organisms.

Considerations of the Hybrid Swarm approach. The Hybrid Swarm approach is 466 applicable to a wide variety of organisms and experimental designs, conferring potential benefits over inbred reference panels. These benefits are realized in three primary ways

468 by: 1) allowing researchers to address questions that require heterozygotes; 2) reducing labor and the influence of block-effects; and 3) breaking down linkage-disequilibrium

470 and population structure that arises from the sampling of individuals from the wild. These benefits are possible due to the ability to accurately reconstruct genomes from ultra-low-coverage sequencing data, i.e. from cost-effective diluted DNA library preparation for a large number of individuals (Baym et al. 2015).

We evaluated simulated Hybrid Swarms founded by 32 or 128 founders to determine 476 the effect of founding haplotype count on reconstruction accuracy and GWAS performance. One might expect 32-founder populations to yield more accurate 478 reconstructions than 128-founder populations, because it is simpler to make a correct inference out of a smaller pool of ancestors. Yet, 32 -founder populations were less

480 accurate in our DGRP simulations (Figure 2), and for some coalescent-based simulations (Supplemental Figure S10). The difference in reconstruction accuracy is

482 perhaps due to the inverse relationship between SNP frequency and information content. In a 128-way cross, rare information-rich SNPs are only likely to exist and be

484 sampled during low-coverage sequencing if nucleotide diversity is high. The DGRP haplotypes happen to exist in a parameter space with higher nucleotide diversity,

486 resulting in 128-way reconstructions being more accurate. For GWAS, 32-founder Hybrid Swarm populations tended to perform better than 128-founder populations if the

488 simulated trait was controlled by a single locus (Figure 5). Taking these two factors into consideration, broadly speaking, a Hybrid Swarm with fewer founding haplotypes may

490 be more appropriate for reconstructing genomes when genetic diversity in the founding lines is low, or if the trait of interest is presumably controlled by one or few loci.

492 Conversely, a greater number of founders may be desired when genetic diversity is high, or the trait of interest is likely to be complex.

The Hybrid Swarm method is not limited to populations founded by inbred lines, as the technique can be applied to populations where phased genomes are available for all outbred founders. Phasing the outbred founders could be accomplished in two basic

498 ways. For instance, phased genomes could be generated using a variety of long-read sequencing technologies (Pollard et al. 2018). Alternatively, trio- and quartet-phasing 500 methods (Patterson et al. 2015), coupled with high coverage sequence data from the 
outbred parents plus a limited $(\leq 2)$ number of F1 offspring, would enable accurate phasing of parents to be used for reconstruction of downstream recombinant genomes.

504 One experimental consideration is the effects of drift and selection that operates during the generation of the Hybrid Swarm (e.g., Thépot et al. (2014)). However, it is not clear

506 that such processes will affect the ability to reconstruct genomes nor the specific outcome of GWAS, unless the trait of interest was under strong selection itself. What

508 may be more likely is the reduction of haplotypic diversity due to drift or selection will slightly decrease power to detect associations, as we see in the differences between

510 32- and 128-way crosses (Supplemental Figure S10). The distribution of haplotypes can also be skewed by line-specific differences in fitness or fecundity, with such differences

512 being observed for DGRP lines (Horváth and Kalinka 2016). To attenuate haplotype dropout, it may be prudent to seed a Hybrid Swarm with a large population of directed

514 F1 hybrids produced by round-robin crosses. The F1 population would then be followed by a limited number of generations (e.g., 4-5) of random outbreeding. This approach

516 was used by Erickson et al (2020) and resulted in a relatively even distribution of founding haplotypes after 5 generations.

We compared genotype calls from our low-coverage reconstruction pipeline to genotype 520 calls from higher coverage data for six experimental Hybrid Swarm individuals (Figure 3 ). Our results show high concordance between reconstructed genomes and high

522 coverage genotypes, particularly if reconstructions with 10 or more inferred recombination events are excluded. Researchers wishing to minimize the impact of

524 inaccurate genotype estimates could implement a stringent limit on inferred recombination counts.

Representation of heterozygotes. One clear difference between inbred and outbred mapping populations is the presence of heterozygotes. On the one hand, the presence of heterozygotes in outbred populations decreases power to detect association relative

530 to inbred lines for an (semi-) additive allele with a given effect size (Figure 5). This basic statistical effect may also be influenced by differences in realized genetic variance

532 between inbred and outbred populations (Genissel et al. 2004). However, the reduced statistical power of association mapping in outbred populations may be ameliorated by

534 reduced inbreeding depression (Lee et al. 2017) and by the ability to assess the heterozygous effects of alleles.

The ability to assess heterozygous effects of alleles will provide valuable insights into several interesting aspects of biology, such as the nature of dominance and the identity of regulatory polymorphisms governing allele-specific expression in a variety of

540 heterogenous genomes. An increased understanding of dominance relationships and regulatory polymorphisms is important for advancing our understanding of quantitative

542 trait variation and evolution. For instance, several theoretical models have shown that context dependent dominance of quantitative fitness traits can underlie the stable

544 maintenance of polymorphisms subject to seasonally variable (Wittmann et al. 2017) or sexually antagonistic (Connallon and Chenoweth 2019) selection. The ability to

546 efficiently map loci with context dependent dominance relationships will aid in the 
548 forms of balancing selection.

550 Regulatory polymorphisms are known to underlie genetic variation in expression (Brem et al. 2002; Cavet et al. 2003; Rockman and Kruglyak 2006) and this expression

552 variation can potentially be resolved to exact nucleotide differences (Grosveld et al. 1987; Rave-Harel et al. 1997; Bosma et al. 2002). The resulting differences in

554 expression can manifest as phenotypic changes to drive local adaptation (Kudaravalli et al. 2009; Fraser et al. 2010; Fraser 2011, 2013). Allele-specific expression (ASE)

556 arising from cis-acting regulatory factors is a common mechanism to produce heritable differences in expression (Yan et al. 2002; Cowles et al. 2002; Lo et al. 2003; Doss

558 2005). Because allelic expression biases are only produced (and detectable) in heterozygotes, Hybrid Swarm populations facilitate the study of regulatory genetic

560 variation (i.e. ASE) as a driver of local adaptation in a variety of organisms.

562 Undirected outbreeding in a common environment. The Hybrid Swarm approach involves propagation of a single large outbred population via undirected crossing. This

564 design confers benefits over alternatives of either rearing inbred lines separately or performing controlled crosses. The relative value of these benefits may vary across

566 organisms and experimental designs.

568 Individuals within a Hybrid Swarm are reared in a common environment. This reduces the influence of random block effects associated with rearing families or closely related

570 individuals in separate enclosures or defined areas. Therefore, studies examining genotype-by-environment interactions can dramatically expand the number of

572 environmental factors used because all individuals were previously reared in a common environment, enabling a Hybrid Swarm to be randomly scatted across environmental

574 treatments. This feature is in contrast to rearing each inbred family across all environments, a practice which generally limits the number of environmental treatments

576 used. Rearing individuals in a common environment also likely reduces genotypeenvironment correlation that could be exacerbated by vial effects.

Random outbreeding of a single population can require less labor compared to

580 performing controlled crosses or serial propagation of inbred lines. The relative value of these benefits is likely to be species specific. For instance, for larger animals (e.g.,

582 mice), controlled crosses may be logistically easier than a randomly mating swarm. For many other species, however, the Hybrid Swarm would be logistically advantageous.

584 The cost of maintaining inbred reference panels also varies among taxa, most notably between those species which can be kept as seeds or in a cryogenically preserved

586 state. For other species, such as flies, the monetary and environmental cost of maintaining hundreds to thousands of inbred lines may be prohibitive.

One drawback draw-back of the Hybrid Swarm is the lack of genetic replicates for

590 phenotyping. Inbred panels allow for a single genotype to be phenotyped multiple times, reducing the effects of error associated with phenotyping and micro-environmental

592 variation (Mackay and Huang 2018). Because this benefit is typically not possible in the 
Hybrid Swarm (unless clones can be propagated), the Hybrid Swarm method

594 represents a tradeoff, reducing the influence of block effects while increasing error associated with phenotyping.

Hybrid Swarm breaks down population structure and linkage disequilibrium.

598 Recombination between lines in the Hybrid Swarm approach allows for greater dissection of functional polymorphisms segregating between genetically structured

600 populations. If an association study incorporates haplotypes from multiple distinct source populations, causal variants would segregate along with other linked variants.

602 Thus, to identify genetic mechanisms of local adaptation and trait variation in general, it is necessary to minimize false positives from linked non-causal loci. Corrections due to

604 relatedness can reduce the type I error rate to some degree (Yu et al. 2006; Price et al. 2010; Yang et al. 2014), and can be further reduced by a greater extent of

606 recombination. Therefore, the Hybrid Swarm approach allows researchers to tailor the mapping population to the traits of interest, by perhaps selecting founders from diverse

608 origins to test specific hypotheses about the distribution of functional variation in the wild.

610

A limited number of rounds of recombination during the propagation of the Hybrid

612 Swarm also reduces long-distance linkage disequilibrium within a panel of inbred lines derived from a single locality. Long-distance LD results from correlated occurrence of

614 rare variants (Huang et al. 2014), potentially contributing to false positives in GWAS (Figure 6). Our simulations showed a genome-wide inflation of $p$-values, even across

616 physically unlinked chromosomes, for inbred panels and this pattern persisted even when rare variants were excluded (Supplemental Figure S13). Two or more generations

618 of recombination were sufficient to reduce this inflation (Figure 6), at least when using association statistics that do not explicitly account for genetic structure in the sample.

620 Whether such long-distance linkage disequilibrium substantially affects the results of GWAS in practice, remains an open question.

Conclusions. Here, we demonstrate the feasibility of genome-reconstruction in Hybrid

624 Swarm populations derived from many founding haplotypes and evaluate the power of this approach for association mapping. Our results suggest that the Hybrid Swarm

626 approach can be a useful alternative to other MPP breeding designs and is applicable to model and non-model organisms.

630 Author Contributions. CW: conceptualization, data curation, methodology, formal analysis, software, validation, visualization, writing the manuscript; ST: investigation, 632 review of manuscript; SR: investigation; AOB: conceptualization, funding acquisition, project administration, resources, supervision, review and editing of the manuscript.

\section{References}


One 10: 1-15.

640 Bosma P. J., J. R. Chowdhury, C. Bakker, S. Gantla, A. de Boer, et al., 2002 The Genetic Basis of the Reduced Expression of Bilirubin UDP-Glucuronosyltransferase

6421 in Gilbert's Syndrome. N. Engl. J. Med. 333: 1171-1175.

644 transcriptional regulation in budding yeast. Science 296: 752-5.

Brem R. B., G. Yvert, R. Clinton, and L. Kruglyak, 2002 Genetic dissection of

$646 \quad$ Sequencing Data

648 gene expression surveyed in maize, mouse and man. Nature 422: 297-302.

650 association studies and the problem of relatedness among advanced intercross lines and other highly recombinant populations. Genetics 185: 1033-1044.

652 Chesler E. J., D. R. Miller, L. R. Branstetter, L. D. Galloway, B. L. Jackson, et al., 2008 The Collaborative Cross at Oak Ridge National Laboratory: Developing a powerful

654 resource for systems genetics. Mamm. Genome 19: 382-389.

656

Chia R., F. Achilli, M. F. W. Festing, and E. M. C. Fisher, 2005 The origins and uses of

mouse outbred stocks. Nat. Genet. 37: 1181-1186.

658 Recombination in Drosophila melanogaster. PLoS Genet. 8: 33-35.

Connallon T., and S. F. Chenoweth, 2019 Dominance reversals and the maintenance of

660

662 genetic variation for fitness. PLoS Biol. 17: 1-11.

Cowles C. R., J. N. Hirschhorn, D. Altshuler, and E. S. Lander, 2002 Detection of

664

Danecek P., A. Auton, G. Abecasis, C. A. Albers, E. Banks, et al., 2011 The variant call format and VCFtools. Bioinformatics 27: 2156-2158.

666

Davies R. W., J. Flint, S. Myers, and R. Mott, 2016 Rapid genotype imputation from

668

Doss S., 2005 Cis-acting expression quantitative trait loci in mice. Genome Res. 15: 681-691.

670

Fraser H. B., A. M. Moses, and E. E. Schadt, 2010 Evidence for widespread adaptive evolution of gene expression in budding yeast. Proc. Natl. Acad. Sci. 107: 29772982.

672 Fraser H. B., 2011 Genome-wide approaches to the study of adaptive gene expression evolution. BioEssays 33: 469-477.

674 Fraser H. B., 2013 Gene expression drives local adaptation in humans. Genome Res. 23: 1089-1096.

676 Genissel A., T. Pastinen, A. Dowell, T. F. C. Mackay, and A. D. Long, 2004 No Evidence for an Association between Common Nonsynonymous Polymorphisms in

678 Delta and Bristle Number Variation in Natural and Laboratory Populations of Drosophila melanogaster. Genetics 166: 291-306.

680 Grosveld F., G. B. van Assendelft, D. R. Greaves, and G. Kollias, 1987 Positionindependent, high-level expression of the human beta-globin gene in transgenic

682 mice. Cell 51: 975-85.

684 for development time and viability in Drosophila melanogaster. Ecol. Evol. 6: 8460- 
8473.

686 Huang B. E., A. W. George, K. L. Forrest, A. Kilian, M. J. Hayden, et al., 2012a A multiparent advanced generation inter-cross population for genetic analysis in wheat. Plant Biotechnol. J. 10: 826-839.

Huang W., S. Richards, M. A. Carbone, D. Zhu, R. R. H. Anholt, et al., 2012b Epistasis dominates the genetic architecture of Drosophila quantitative traits. Proc. Natl. Acad. Sci. 109: 15553-15559.

Huang W., A. Massouras, Y. Inoue, J. Peiffer, M. Ramia, et al., 2014 Natural variation in genome architecture among 205 Drosophila melanogaster Genetic Reference

694 Panel lines. Genome Res. 24: 1193-1208.

Huang W., T. Campbell, M. A. Carbone, W. E. Jones, D. Unselt, et al., 2020 Contextdependent genetic architecture of Drosophila life span. PLoS Biol. 18: e3000645.

Kessner D., T. L. Turner, and J. Novembre, 2013 Maximum likelihood estimation of frequencies of known haplotypes from pooled sequence data. Mol. Biol. Evol. 30: 1145-58.

700 King E. G., C. M. Merkes, C. L. McNeil, S. R. Hoofer, S. Sen, et al., 2012 Genetic dissection of a model complex trait using the Drosophila Synthetic Population

702 Resource. Genome Res. 22: 1558-1566.

704

Kover P. X., W. Valdar, J. Trakalo, N. Scarcelli, I. M. Ehrenreich, et al., 2009 A Multiparent Advanced Generation Inter-Cross to Fine-Map Quantitative Traits in Arabidopsis thaliana, (R. Mauricio, Ed.). PLoS Genet. 5: e1000551.

706

Krämer N., N. Ranc, N. Meyer, M. Ouzunova, C. Lehermeier, et al., 2014 Usefulness of Multiparental Populations of Maize ( Zea mays L.) for Genome-Based Prediction .

708 Genetics 198: 3-16.

Kudaravalli S., J. B. Veyrieras, B. E. Stranger, E. T. Dermitzakis, and J. K. Pritchard, 2009 Gene expression levels are a target of recent natural selection in the human genome. Mol. Biol. Evol. 26: 649-658.

712

Lack J. B., C. M. Cardeno, M. W. Crepeau, W. Taylor, R. B. Corbett-Detig, et al., 2015 The drosophila genome nexus: A population genomic resource of 623 Drosophila melanogaster genomes, including 197 from a single ancestral range population. Genetics 199: 1229-1241.

Lander E. S., and S. Botstein, 1989 Mapping mendelian factors underlying quantitative traits using RFLP linkage maps. Genetics 121: 185.

718 Lee Y. C. G., Q. Yang, W. Chi, S. A. Turkson, W. A. Du, et al., 2017 Genetic architecture of natural variation underlying adult foraging behavior that is essential for survival of Drosophila melanogaster. Genome Biol. Evol. 9: 1357-1369.

722

Li R., M. A. Lyons, H. Wittenburg, B. Paigen, and G. A. Churchill, 2005 Combining data from multiple inbred line crosses improves the power and resolution of quantitative trait loci mapping. Genetics 169: 1699-709.

724 Li H., B. Handsaker, A. Wysoker, T. Fennell, J. Ruan, et al., 2009 The Sequence Alignment/Map format and SAMtools. Bioinformatics 25: 2078-2079.

726 Li H., 2011 wgsim (short read simulator). GitHub repository: https://github.com/lh3/wgsim

728 Li H., 2013 Aligning sequence reads, clone sequences and assembly contigs with BWAMEM

730 Lo H. S., Z. Wang, Y. Hu, H. H. Yang, S. Gere, et al., 2003 Allelic variation in gene 
expression is common in the human genome. Genome Res. 13: 1855-62.

Long A. D., and C. H. Langley, 1999 The power of association studies to detect the contribution of candidate genetic loci to variation in complex traits. Genome Res. 9: 720-731.

Long A. D., S. J. Macdonald, and E. G. King, 2014 Dissecting complex traits using the Drosophila Synthetic Population Resource. Trends Genet. 30: 488-495.

Mackay T. F. C., and W. Huang, 2018 Charting the genotype-phenotype map: lessons from the Drosophila melanogaster Genetic Reference Panel. Wiley Interdiscip. Rev. Dev. Biol. 7: e289.

MacKay T. F. C. C., S. Richards, E. A. Stone, A. Barbadilla, J. F. Ayroles, et al., 2012 The Drosophila melanogaster Genetic Reference Panel. Nature 482: 173-178.

742 Marigorta U. M., J. A. Rodríguez, G. Gibson, and A. Navarro, 2018 Replicability and Prediction: Lessons and Challenges from GWAS. Trends Genet. 34: 504-517.

744 Monir M. M., and J. Zhu, 2017 Comparing GWAS Results of Complex Traits Using Full Genetic Model and Additive Models for Revealing Genetic Architecture. Sci. Rep. 7: 38600.

748

Mott R., C. J. Talbot, M. G. Turri, A. C. Collins, and J. Flint, 2000 A method for fine mapping quantitative trait loci in outbred animal stocks. Proc. Natl. Acad. Sci. 97: 12649-54.

752

Nikpay M., A. Goel, H. H. Won, L. M. Hall, C. Willenborg, et al., 2015 A comprehensive 1000 Genomes-based genome-wide association meta-analysis of coronary artery disease. Nat. Genet. 47: 1121-1130.

754

Patterson M., T. Marschall, N. Pisanti, L. van lersel, L. Stougie, et al., 2015 WhatsHap: Weighted Haplotype Assembly for Future-Generation Sequencing Reads. J. Comput. Biol. 22: 498-509.

756 Picard toolkit, 2019 Broad Institute, GitHub Repository: https://github.com/broadinstitute/picard; Broad Institute.

758 Pollard M. O., D. Gurdasani, A. J. Mentzer, T. Porter, and M. S. Sandhu, 2018 Long reads: their purpose and place. Hum. Mol. Genet. 27: R234-R241.

760 Price A. L., N. A. Zaitlen, D. Reich, and N. Patterson, 2010 New approaches to population stratification in genome-wide association studies. Nat. Rev. Genet. 11:

762 459-463.

R Core Team, 2016 R: A Language and Environment for Statistical Computing

764 Rave-Harel N., E. Kerem, M. Nissim-Rafinia, I. Madjar, R. Goshen, et al., 1997 The molecular basis of partial penetrance of splicing mutations in cystic fibrosis. Am. J.

$766 \quad$ Hum. Genet. 60: 87-94.

Reich D. E., and D. B. Goldstein, 2000 Detecting association in a case-control study

768 while correcting for population stratification. Genet. Epidemiol. 20: 4-16.

770

Rockman M. V., and L. Kruglyak, 2006 Genetics of global gene expression. Nat. Rev. Genet. 7: 862-872.

772

Rockman M. V., and L. Kruglyak, 2008 Breeding designs for recombinant inbred advanced intercross lines. Genetics 179: 1069-1078.

Singh R., I. T. Lobina, M. Thomson, S. McCouch, C. Dilla-Ermita, et al., 2013 Multi-

774 parent advanced generation inter-cross (MAGIC) populations in rice: progress and potential for genetics research and breeding. Rice 6: 11.

776 Spencer C. C. A., Z. Su, P. Donnelly, and J. Marchini, 2009 Designing genome-wide 
association studies: Sample size, power, imputation, and the choice of genotyping chip. PLoS Genet. 5.

Staab P. R., S. Zhu, D. Metzler, and G. Lunter, 2015 scrm: efficiently simulating long sequences using the approximated coalescent with recombination. Bioinformatics 31: 1680-1682.

782 Stevenson M., 2018 epiR: Tools for the Analysis of Epidemiological Data

Svenson K. L., D. M. Gatti, W. Valdar, C. E. Welsh, R. Cheng, et al., 2012 Highresolution genetic mapping using the mouse Diversity Outbred population. Genetics 190: 437-447.

Thépot S., G. Restoux, I. Goldringer, F. Hospital, D. Gouache, et al., 2014 Efficiently tracking selection in a multiparental population: The case of earliness in wheat. Genetics 199: 609-623.

Turck N., L. Vutskits, P. Sanchez-Pena, X. Robin, A. Hainard, et al., 2011 pROC: an open-source package for $\mathrm{R}$ and $\mathrm{S}+$ to analyze and compare $\mathrm{ROC}$ curves. BMC Bioinformatics 8: 12-77.

792 Valdar W., J. Flint, and R. Mott, 2006 Simulating the collaborative cross: Power of quantitative trait loci detection and mapping resolution in large sets of recombinant inbred strains of mice. Genetics 172: 1783-1797.

Wittmann M. J., A. O. Bergland, M. W. Feldman, P. S. Schmidt, and D. A. Petrov, 2017 Seasonally fluctuating selection can maintain polymorphism at many loci via segregation lift. Proc. Natl. Acad. Sci. 114: E9932-E9941.

Wu Y., Z. Zheng, P. M. Visscher, and J. Yang, 2017 Quantifying the mapping precision of genome-wide association studies using whole-genome sequencing data.

$800 \quad$ Genome Biol. 18: 1-10.

Xu S., and W. R. Atchley, 1996 Mapping quantitative trait loci for complex binary diseases using line crosses. Genetics 143: 1417-1424.

Yan H., W. Yuan, V. E. Velculescu, B. Vogelstein, and K. W. Kinzler, 2002 Allelic

$804 \quad$ variation in human gene expression. Science 297: 1143.

806 factors under polygenic inheritance. Eur. J. Hum. Genet. 19: 807-812.

Yang J., N. A. Zaitlen, M. E. Goddard, P. M. Visscher, and A. L. Price, 2014 Advantages

808 and pitfalls in the application of mixed-model association methods. Nat. Genet. 46: 100-106.

810 Yu J., G. Pressoir, W. H. Briggs, I. V. Bi, M. Yamasaki, et al., 2006 A unified mixedmodel method for association mapping that accounts for multiple levels of

812 relatedness. Nat. Genet. 38: 203-208.

Zhang J., K. Kobert, T. Flouri, and A. Stamatakis, 2014 PEAR: A fast and accurate

814 Illumina Paired-End reAd mergeR. Bioinformatics 30: 614-620.

Zheng C., M. P. Boer, and F. A. van Eeuwijk, 2015a Reconstruction of genome ancestry

816 blocks in multiparental populations. Genetics 200: 1073-1087.

Zheng C., M. P. Boer, and F. A. van Eeuwijk, 2015b Reconstruction of genome ancestry

818 blocks in multiparental populations. Genetics 200: 1073-1087.

Zheng C., M. P. Boer, and F. A. van Eeuwijk, 2018 Accurate genotype imputation in

820 multiparental populations from low-coverage sequence. Genetics 210: 71-82. 
Figures and Figure Legends

Founding Diversity Breeding Schema Haplotype Structure

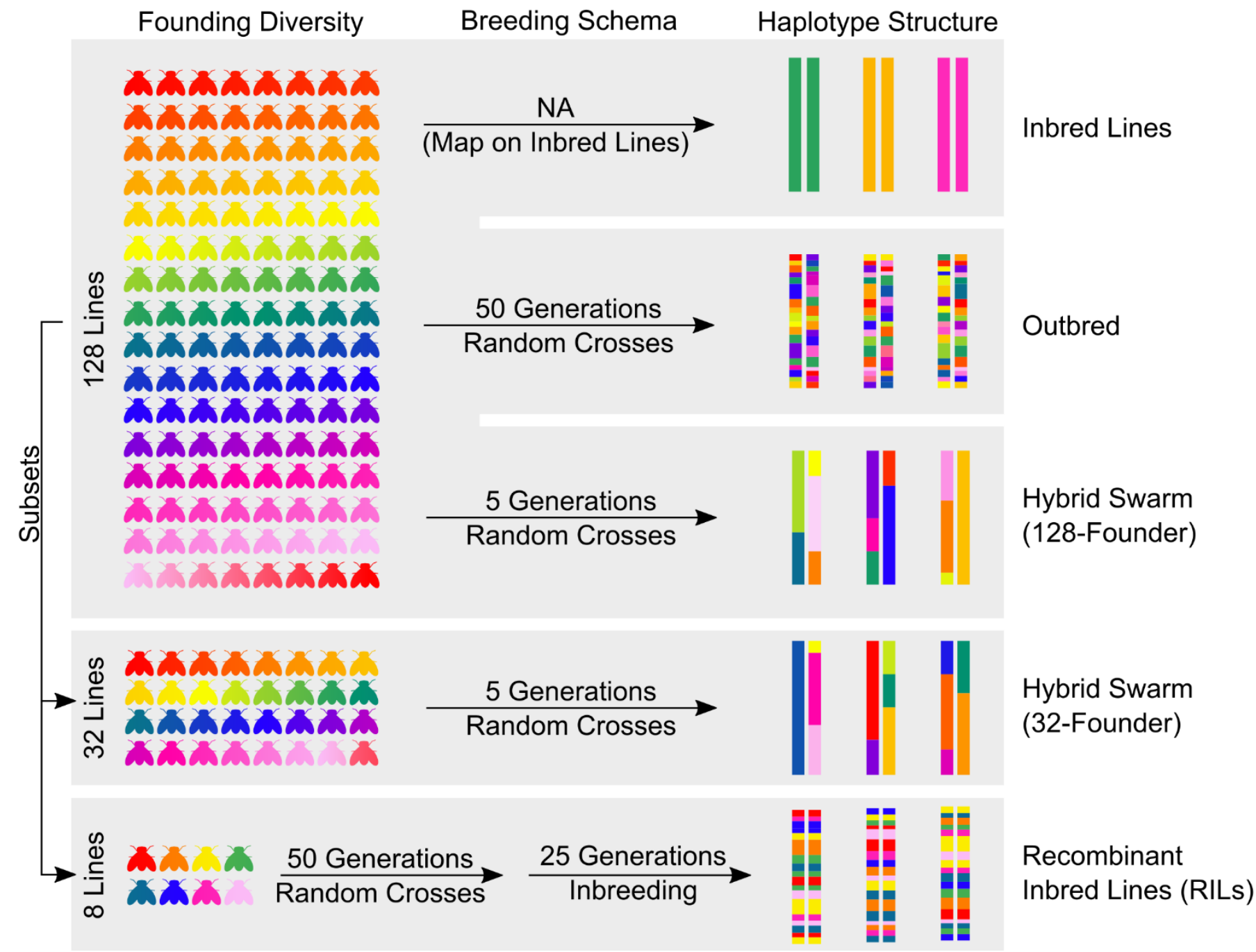

Figure 1. Depictions of various mapping populations explored. Each color represents a unique haplotype. 


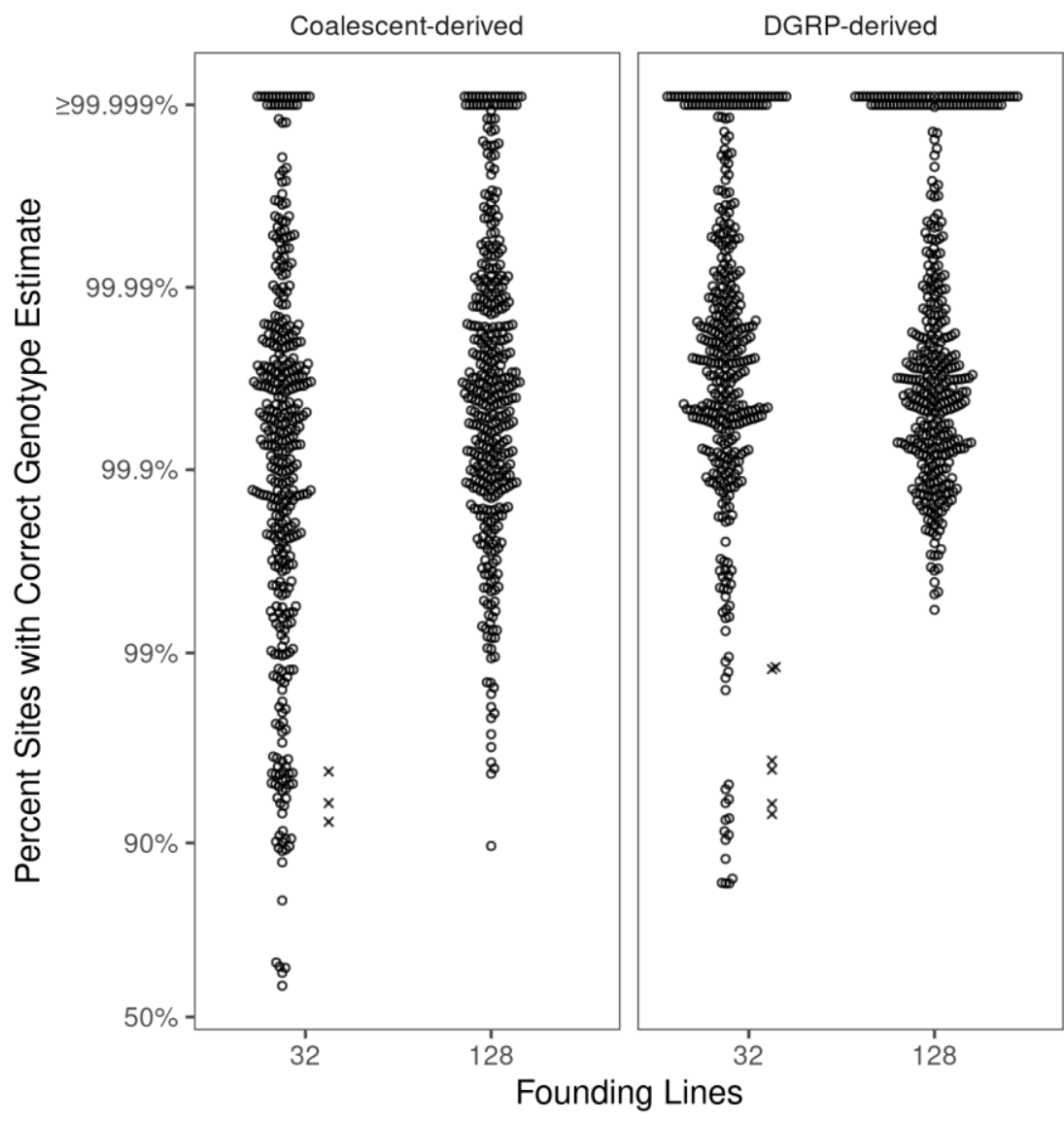

Hyper-recombinant

- FALSE

$\times$ TRUE

Figure 2. Genotype estimation accuracy for Hybrid Swarm populations.

830 Reconstructions were performed for populations simulated using 32 or 128 inbred founder. Accuracy, calculated as the per-chromosome fraction of variable sites with a

832 correct diploid genotype estimate, is shown on logit-transformed scale. Values are coded depending on the number of estimated recombination events, with highly

834 recombinant estimates (>10 recombination events) displayed as an $\times$. Each parameter combination includes 400 reconstructed autosomes (individual circles) for 100 simulated

836 individuals. The coalescent-derived individuals displayed here were simulated with an effective population size of $\mathrm{N}_{\mathrm{e}}=1 \times 10^{6}$ and mutation rate $\mu=5 \times 10^{-9}$. 
A

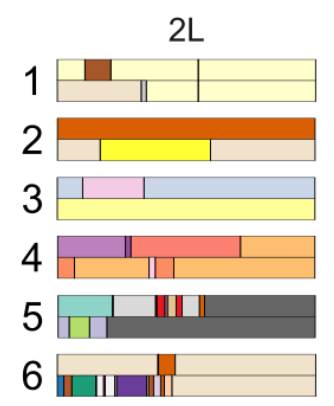

2R

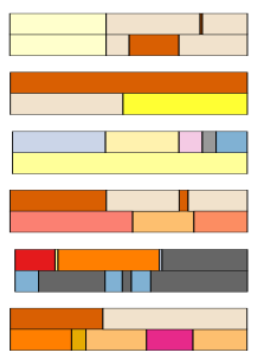

3L

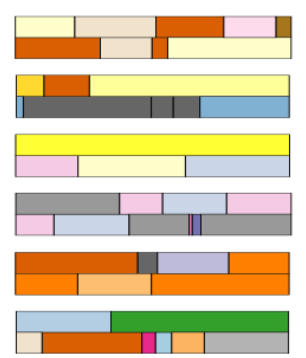

$3 R$

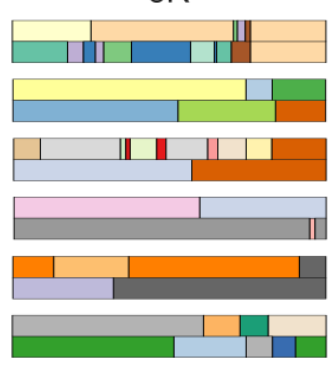

X

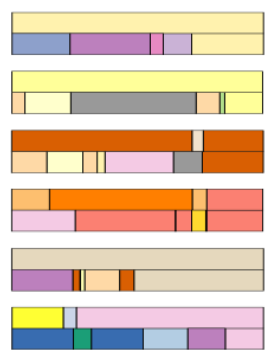

\section{B}

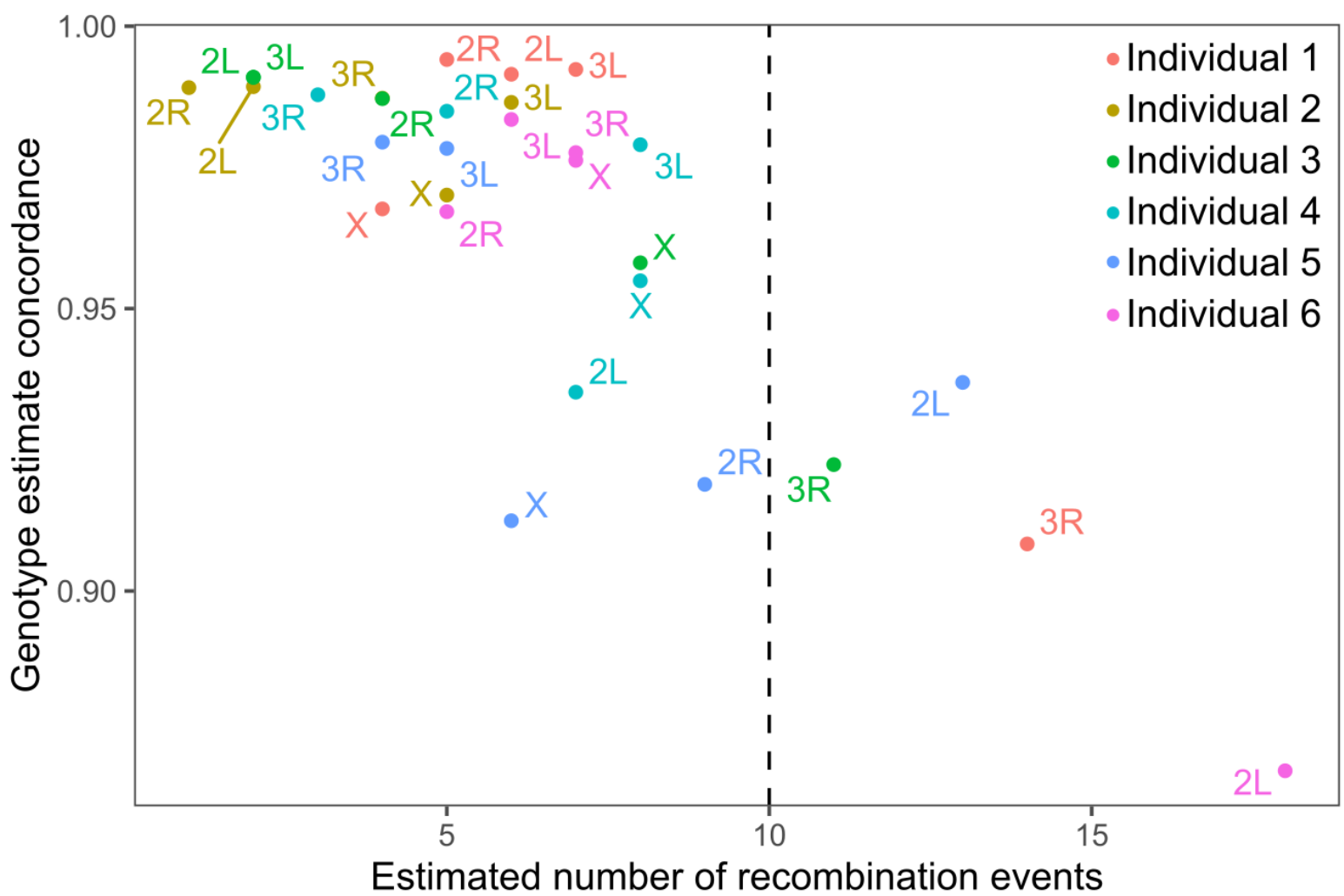

840 Figure 3. RABBIT chromosome reconstructions for real-life hybrid swarm individuals. A: Depictions of estimated haplotypes for six low-coverage $F_{5}$ Drosophila

842 melanogaster, identified by sample number. B: Genotype estimate concordance between our reconstruction pipeline and higher-coverage genotype calls. Colors

844 correspond to individuals 1-6 in panel A. Our low-coverage genotype estimates tend to be $97-99 \%$ concordant with high-coverage calls, except in cases where RABBIT

846 estimates are highly recombinant. A dashed line at 10 recombination events marks our threshold for excluding assumedly inaccurate hyper-recombinant reconstructions. 

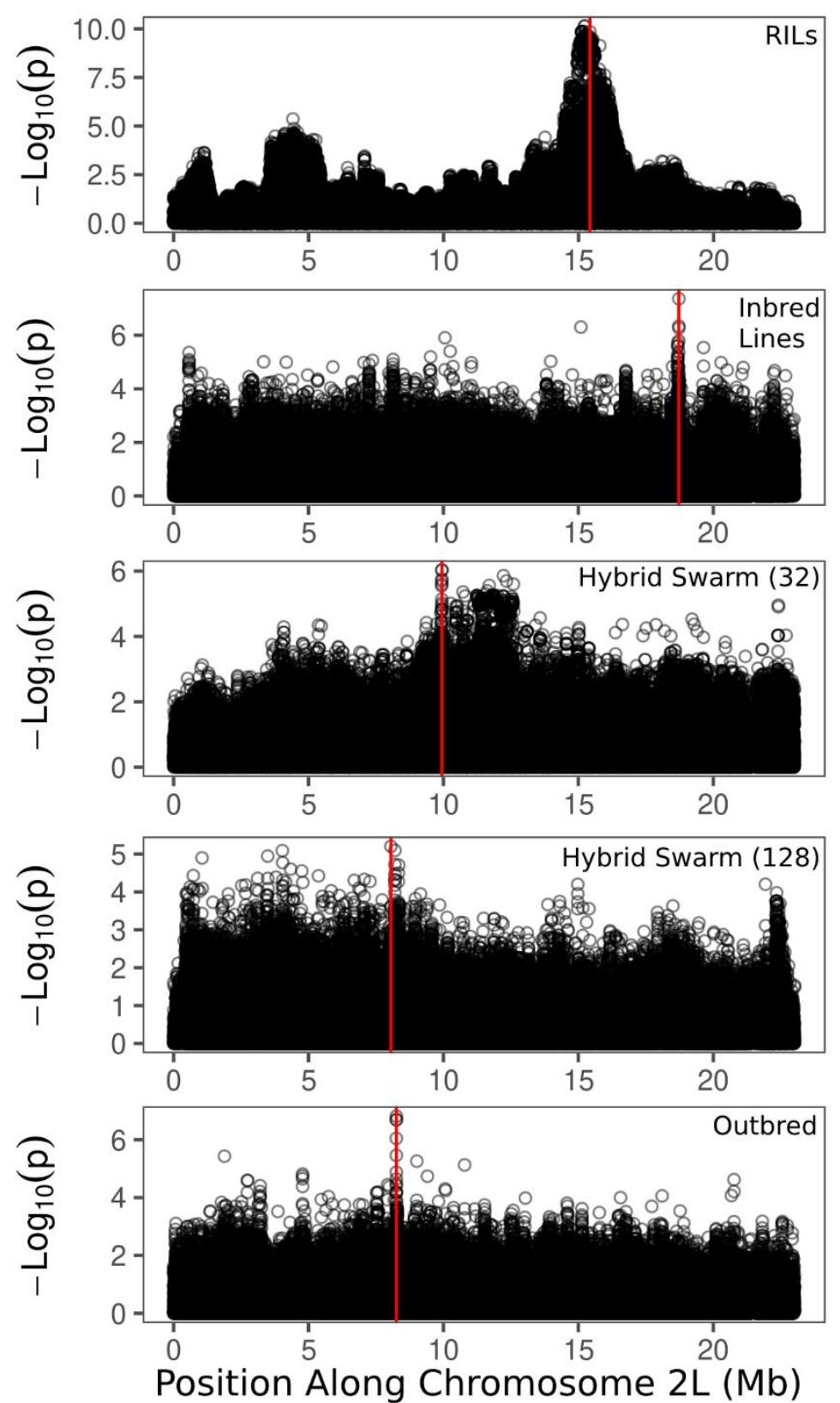

850 Figure 4. Examples of Manhattan plots from our simulated GWAS. These simulations correspond to a single causal locus of large effect, with position indicated by the vertical 852 red line. Recombinant Inbred Lines tend to display a wide association peak near the causal locus, while other populations show only a small number of sites tightly clustered 854 on the causal locus. 


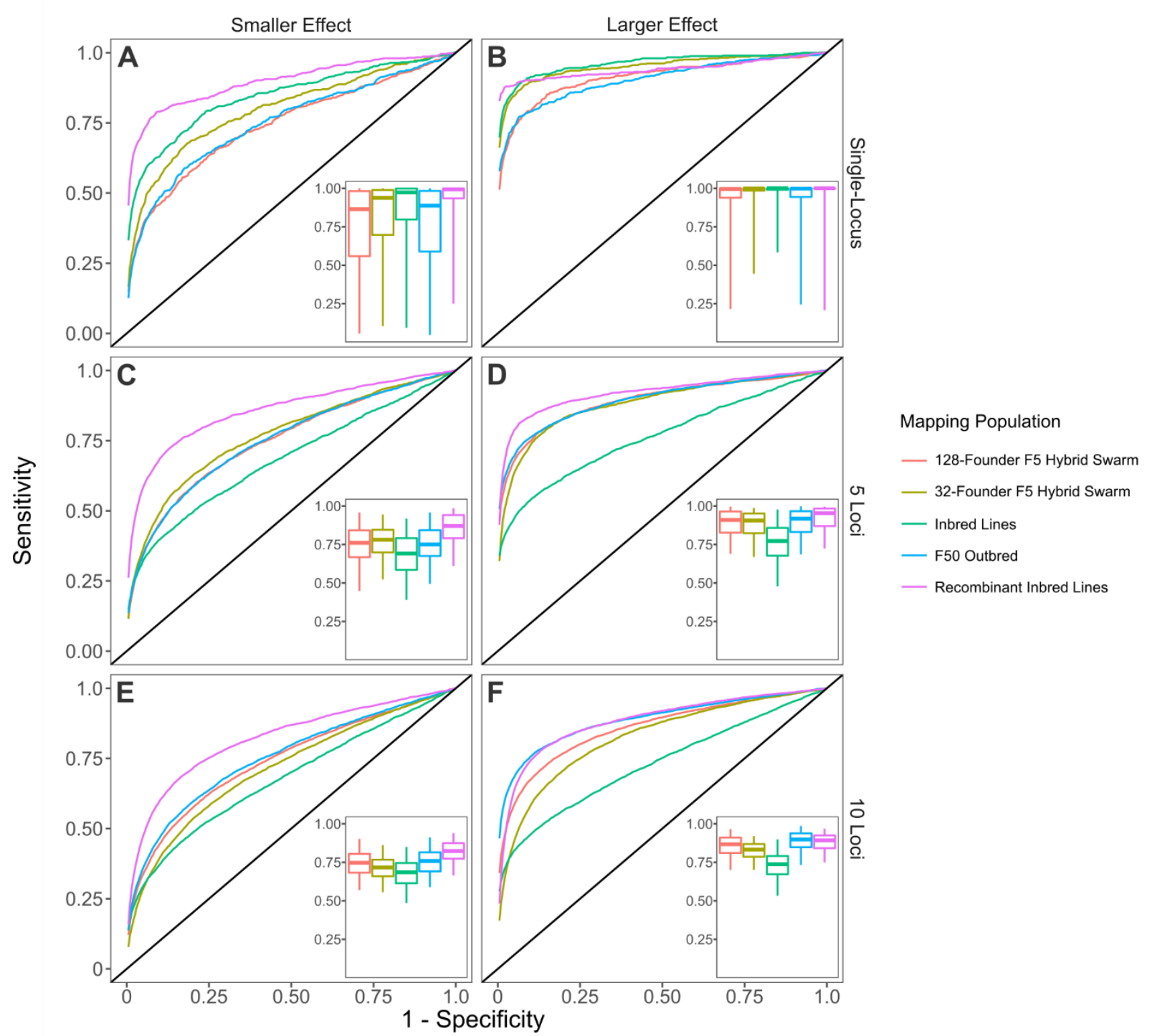

856 Figure 5. Receiver Operator Characteristic (ROC) curves for GWAS simulations. ROC curves represent the aggregate of 500 GWAS simulations (each comprised of 8585000 individuals) per parameter combination. To generate single representative ROC curves for each population, we stepped through all specificity values and calculated the

860 mean sensitivity. Inset boxplots display the distribution of AUCs, with whiskers spanning the middle $95 \%$ of data. See methods for descriptions for generating each mapping

862 population. 

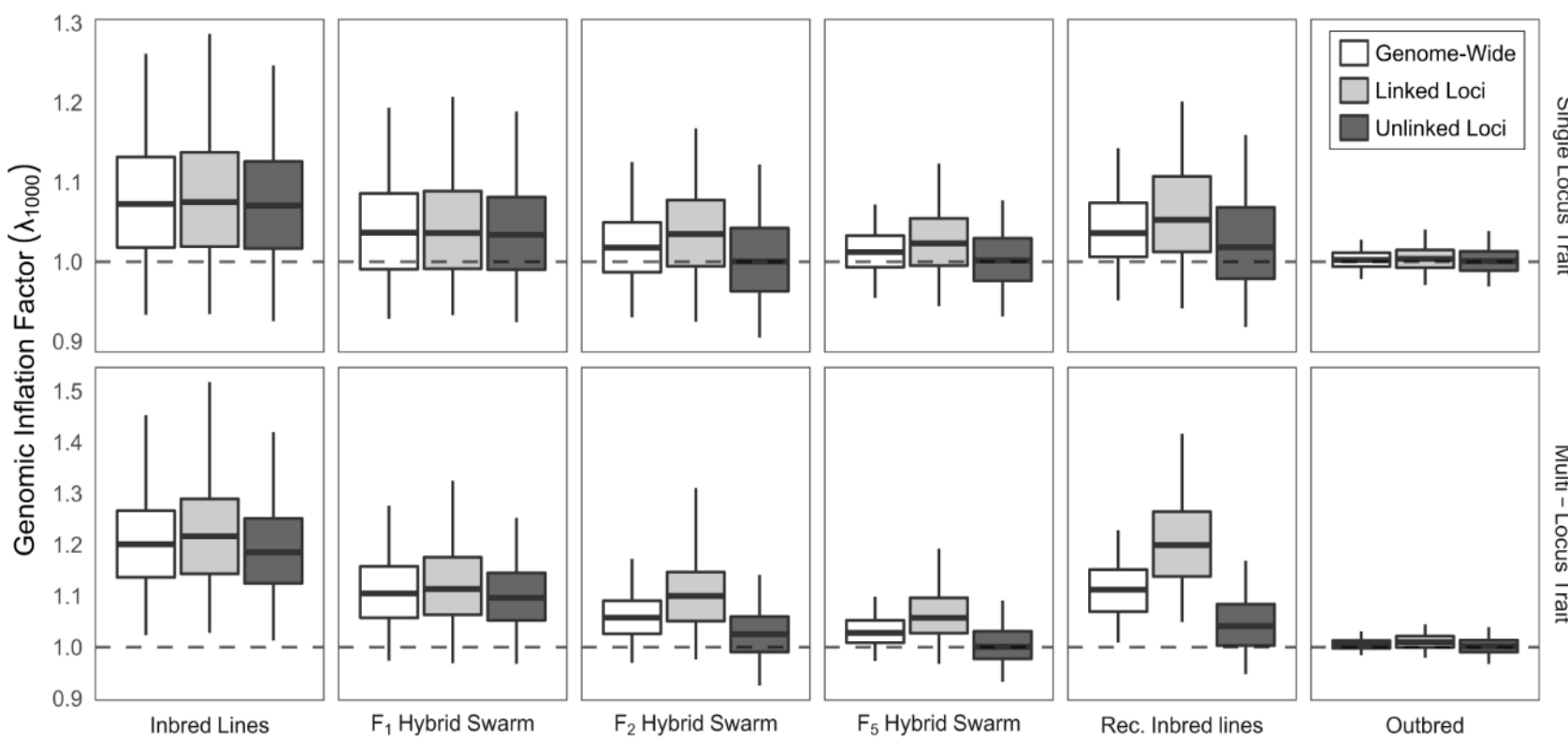

Mapping Population

Figure 6. Genomic Inflation Factor $\left(\mathrm{GIF}, \lambda_{1000}\right)$ for simulated GWAS with monogenic trait

866 of with a large effect locus, or multi-locus trait of smaller individual effects.

GIF is calculated genome-wide (across all autosomes); on the autosome arm containing

868 the causal allele (linked); and for sites on the autosome physically unlinked to the causal allele. $\lambda$ is calculated as the ratio of observed to expected $x 2$ values, and a

870 correction is performed to produce the null expectation of a sample size equal to 1000 individuals (see Materials and Methods for details). Data are averaged over 500 GWAS

872 simulations (each comprised of 5000 individuals), with phenotypes assigned in a casecontrol framework. Boxes represent the median and interquartile range; whiskers

874 extending to the lower and upper bounds of the 95\% quantiles. Hybrid Swarm populations here used 128 founders. Outbred: F50 population founded by 128 inbred 876 lines. 


$\begin{array}{ccccc}\text { Population } & \text { N Founders } & \rho & \text { Mean } \Delta & \sigma_{\Delta} \\ \text { DGRP } & 128 & 0.986 & -0.015 & 0.25 \\ \text { DGRP } & 32 & 0.502 & 0.17 & 2.15 \\ \text { Coalescent } & 128 & 0.956 & -0.17 & 0.44 \\ \text { Coalescent } & 32 & 0.759 & -0.31 & 1.26\end{array}$

Table 1. Accuracy of estimated number of recombination events following chromosome 880 reconstruction from $0.05 \mathrm{X}$ sequencing data. A high concordance correlation coefficient (Lin's $\rho$ ) indicates agreement between estimated and true recombination counts for 400

882 reconstructed chromosomes (coalescent-derived populations) or chromosome arms (DGRP-derived populations). Coalescent-derived populations are described across a

884 range of values for effective population size $\mathrm{N}_{\mathrm{e}}$ and mutation rate $\mu . \Delta$ represents the difference between estimated and true recombination counts, and $\sigma_{\Delta}$ represents the

886 mean of 400 standard deviations of $\Delta$. Reconstructions were performed with a maximum of 16 most-likely-ancestors with a HARP threshold of 0.99 (see methods for

888 more details). The coalescent-derived populations described here were simulated with an effective population size of $\mathrm{Ne}_{\mathrm{e}}=1 \times 10^{6}$ and mutation rate $\mu=5 \times 10^{-9}$. 


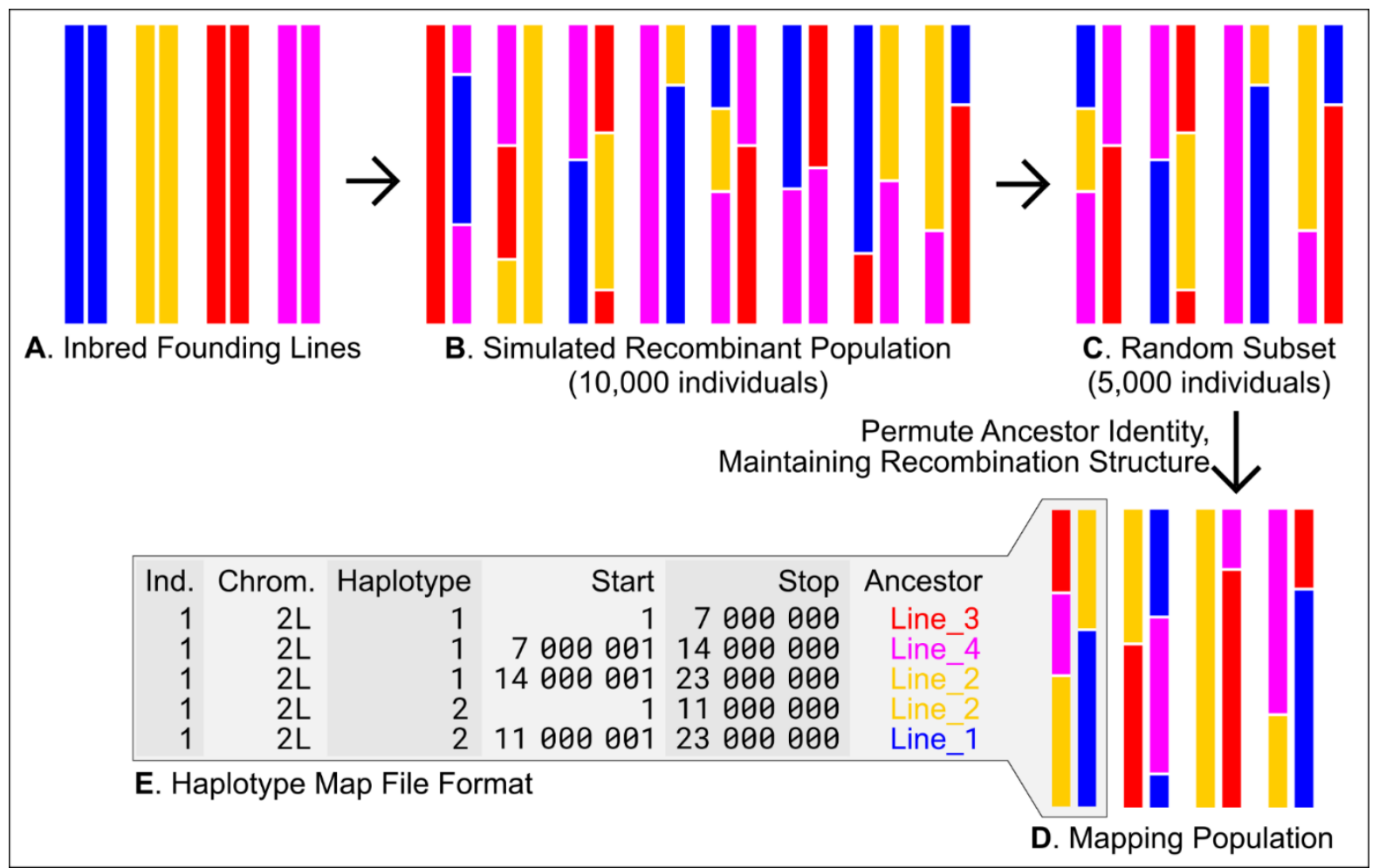

892 Supplemental Figure S1. Basic structure of the forward simulator pipeline. Inbred founding lines $(\mathbf{A})$ are randomly intercrossed to produce a recombinant

894 population (B). Rapid generation of independent mapping populations is achieved by random down-sampling (C) and permutation of ancestry (D). Population genetic data is

896 encoded in a highly compressed format (E) that references the positions of haplotype blocks instead of genotypes at every site, enabling us to generate 500 mapping

898 populations for a given parameter combination. 

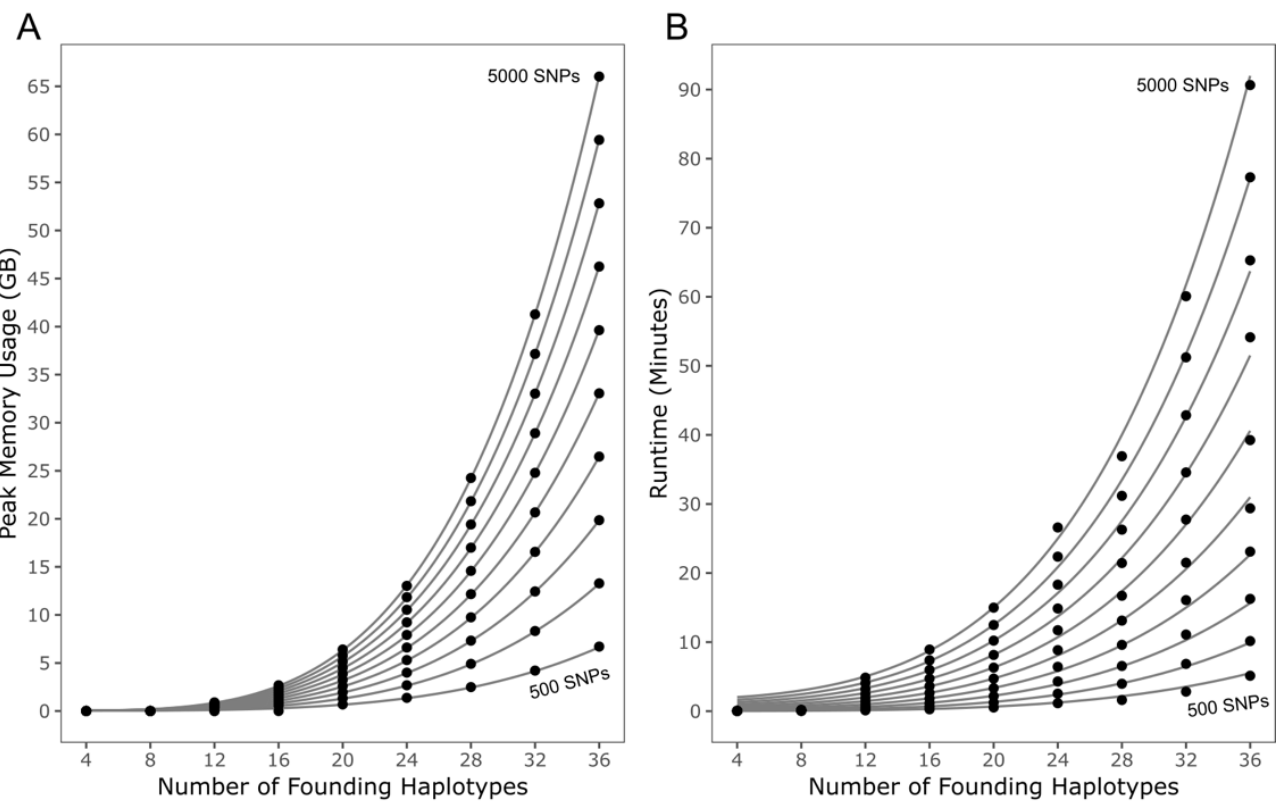

C

D
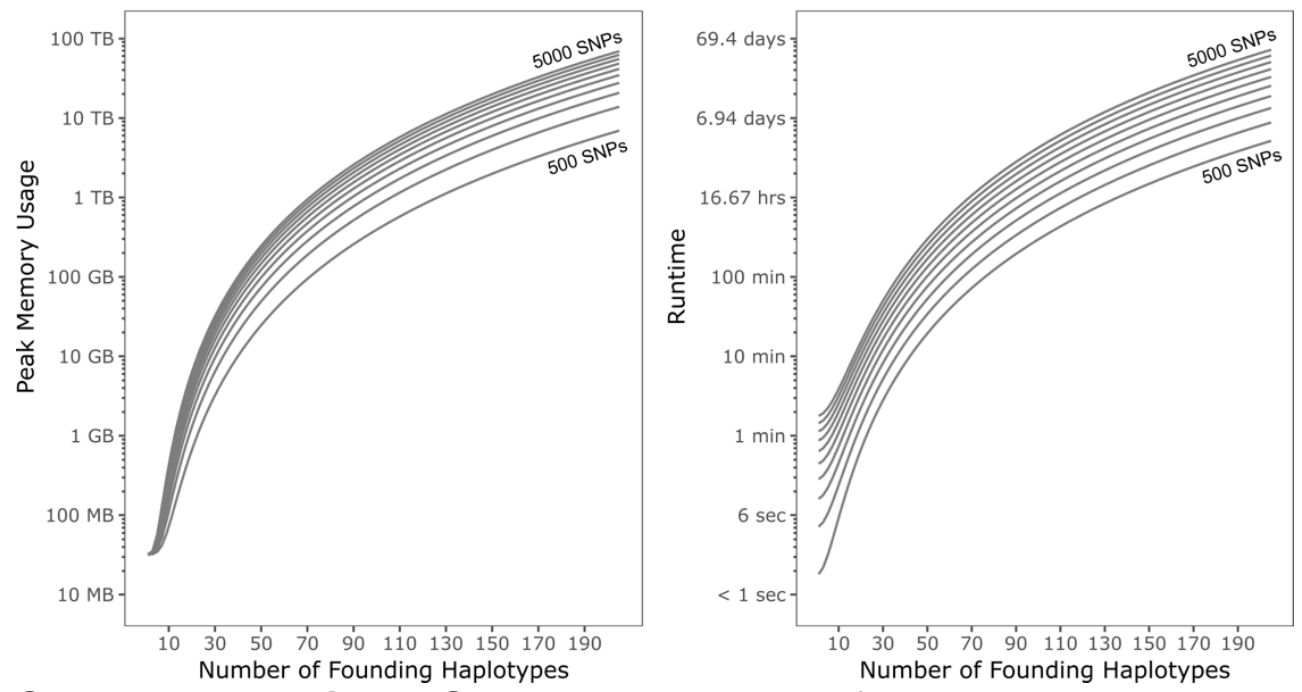

Supplemental Figure S2. Resource usage of RABBIT during haplotype reconstruction.

902 All reconstructions involve the same simulated $2 \mathrm{~L}$ chromosome arm comprised of four haplotypes. Simulations included varied numbers of founding haplotypes $(N)$ and a

904 randomly selected set of markers (number of SNPs, S, incremented in steps of 500). All simulations included, at minimum, the four true haplotypes for the simulated individual.

906 In $\mathbf{A}$ and $\mathbf{B}$, points depict the mean of empirical values (over 10 replicates) and gray lines depict the defined regression models. Regression models for memory usage and

908 runtime are extrapolated over a greater range for number of founding haplotypes in $\mathbf{C}$ and $\mathbf{D}$, respectively. Peak memory grew linearly with number of SNPs used, and at a

910 greater-than-linear rate with haplotypes (A). The runtime of RABBIT increased at a greater-than-linear rate for both number of SNPs and number of haplotypes, with the N

912 parameter contributing most to resource cost (B). These models allowed us to estimate resource requirements at greater numbers of haplotypes $(C \& D)$ which would be

914 unfeasible to measure empirically. 
bioRxiv preprint doi: https://doi.org/10.1101/671925; this version posted January 27, 2021. The copyright holder for this preprint (which was not certified by peer review) is the author/funder, who has granted bioRxiv a license to display the preprint in perpetuity. It is made available under aCC-BY-NC-ND 4.0 International license.

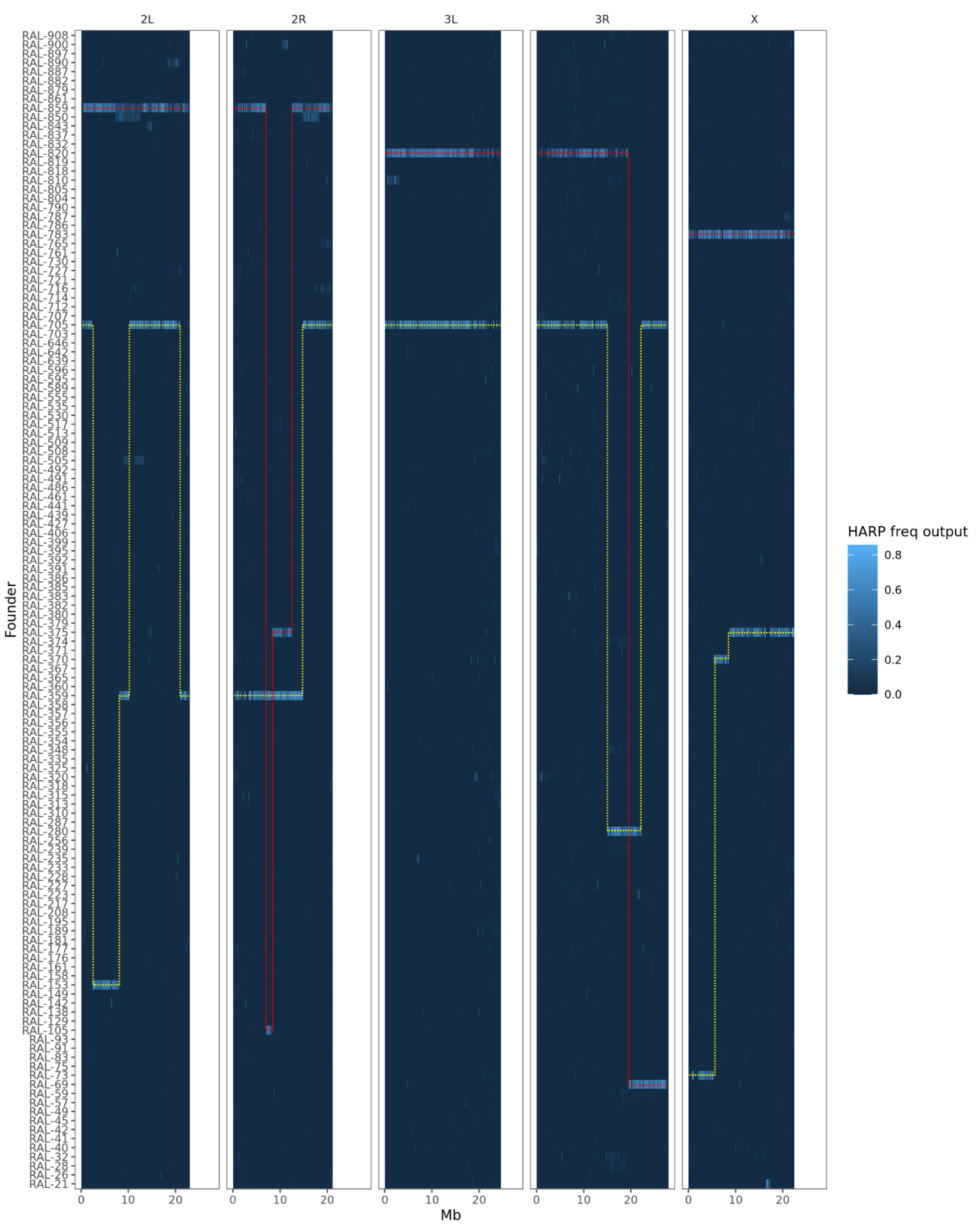

916 Supplemental Figure S3. Example of unambiguous haplotype inference from HARP. For each chromosome, each haplotype is indicated by red and yellow dotted lines. 


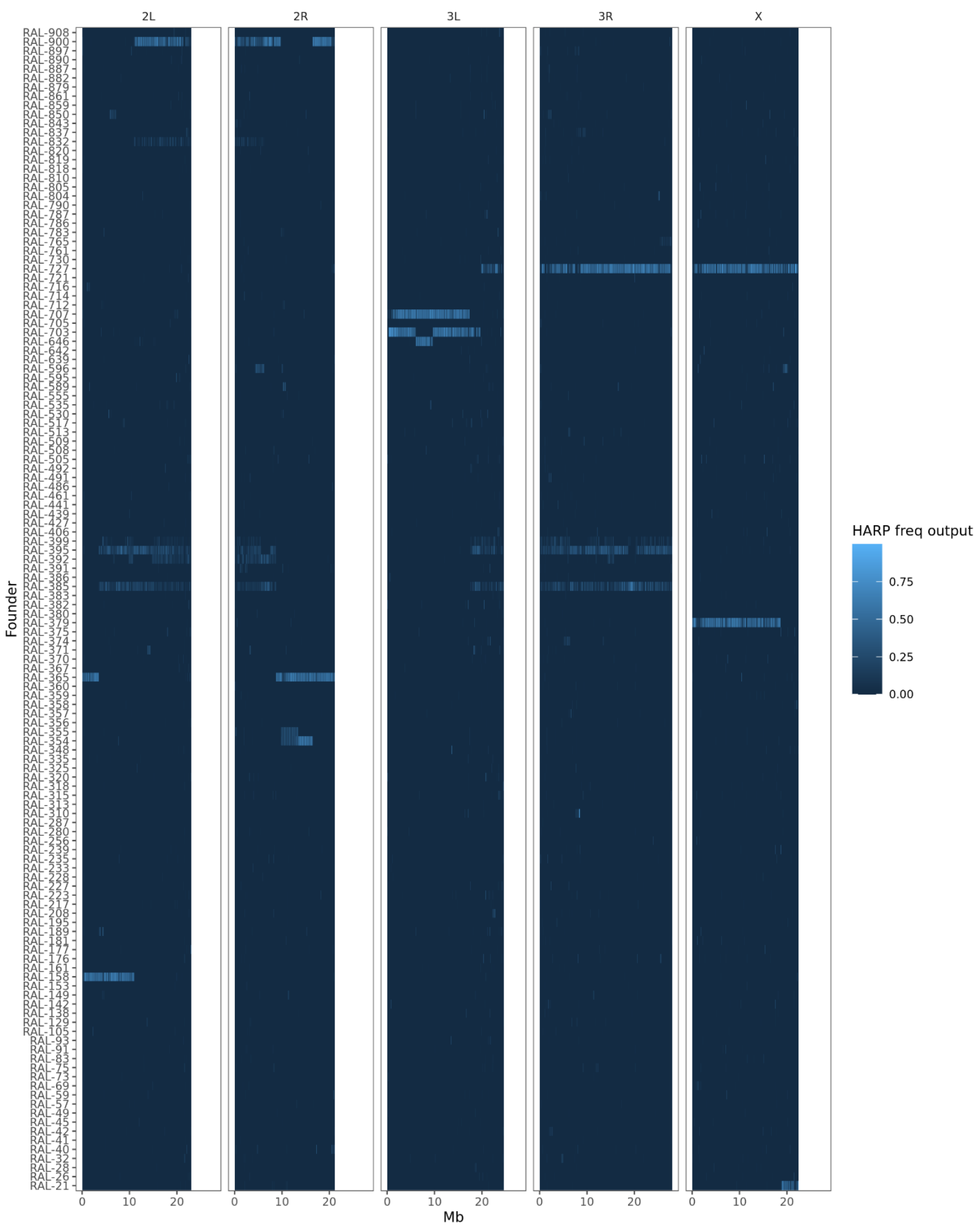

Supplemental Figure S4. Example of HARP freq output with ambiguous haplotype 920 inference. For each chromosome, each haplotype is indicated by red and yellow dotted lines. For this individual's autosomes, at least one haplotype cannot be unambiguously 922 resolved. 


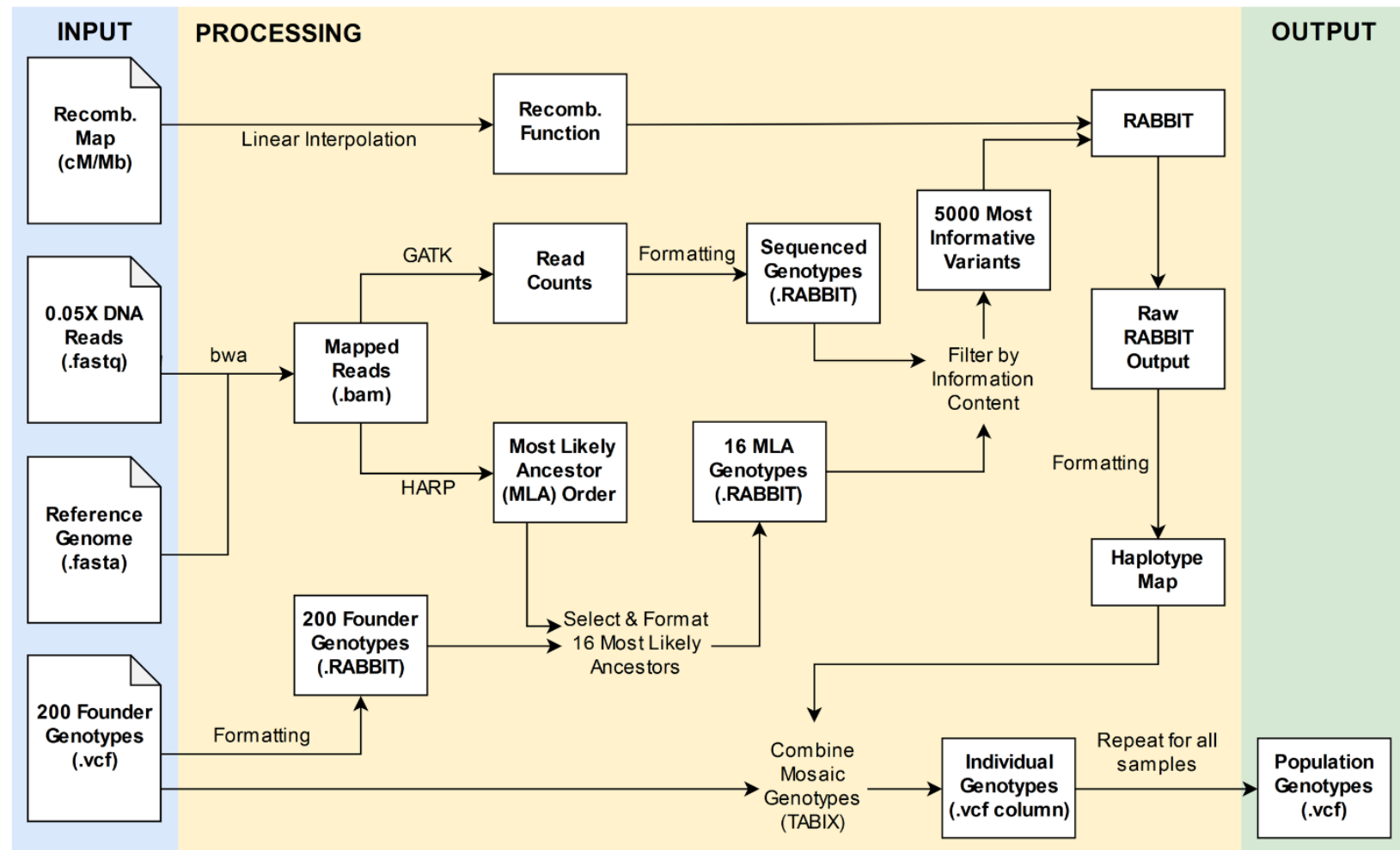

Supplemental Figure S5. Workflow for reconstructing whole genomes from ultra-low 926 coverage sequencing data. Three required inputs are sequencing reads (.fastq), a reference genome (.fasta), and homozygous (or phased) founder haplotypes (.vcf file).

928 A recombination map can optionally be provided for use with RABBIT to inform variation in recombination rates across chromosomes. Flowchart designed in draw.io. 

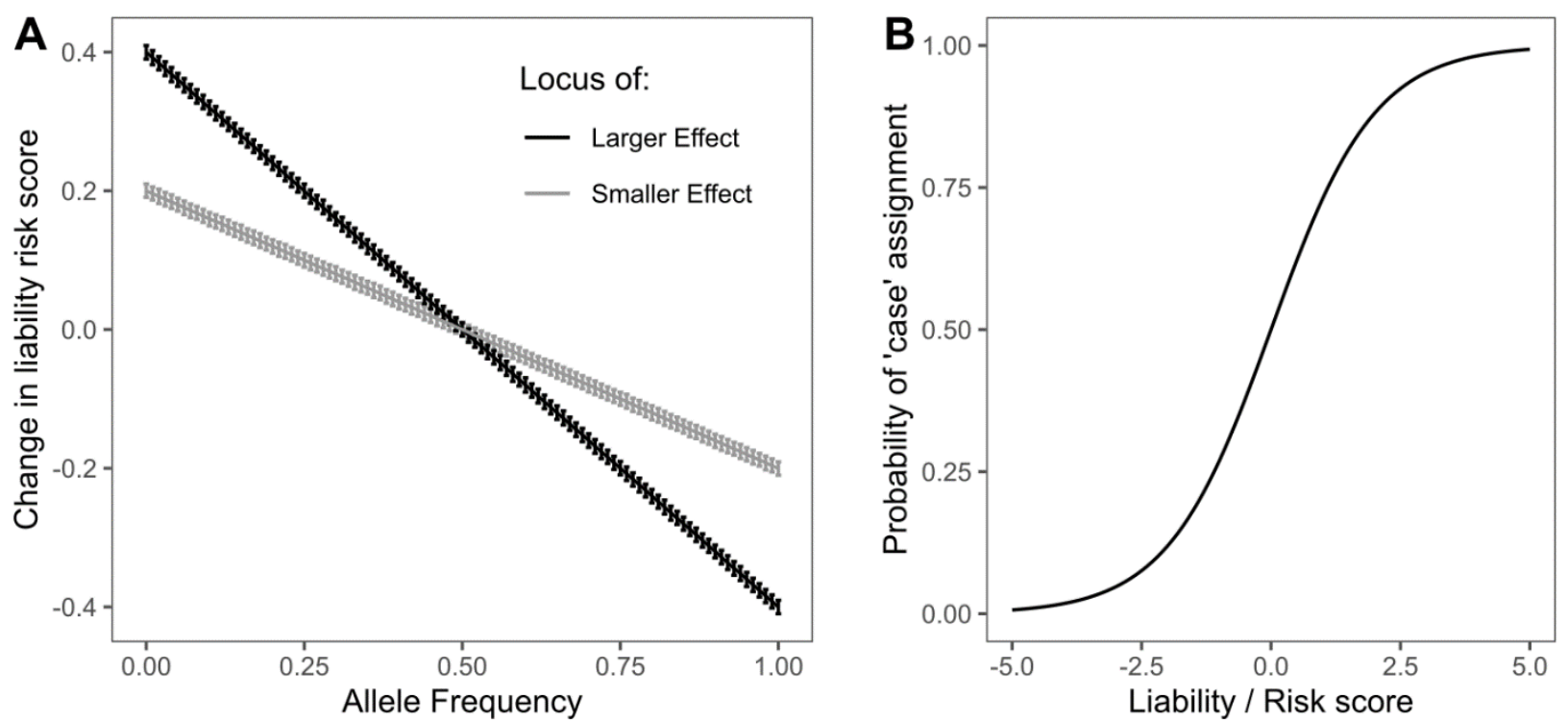

Supplemental Figure S6. Liability phenotype model for GWAS simulations. for

934 transforming additive risk scores to probability of assignment to the case population, i.e. probability of developing a disease. Rare alleles increase risk score, which in turns

936 increases probability of being assigned a case phenotype. A: The relationship between allele frequency and effect on liability risk score. Simulations were modeled as larger or

938 smaller effect with a user-defined effect size coefficient. Error bars describe the standard deviation of a gaussian sample which constitutes random phenotypic noise. B:

940 Logistic transformation of the form $y=\left(1+e^{-x}\right)^{-1}$ translates the liability score into probability of being assigned to case population. 


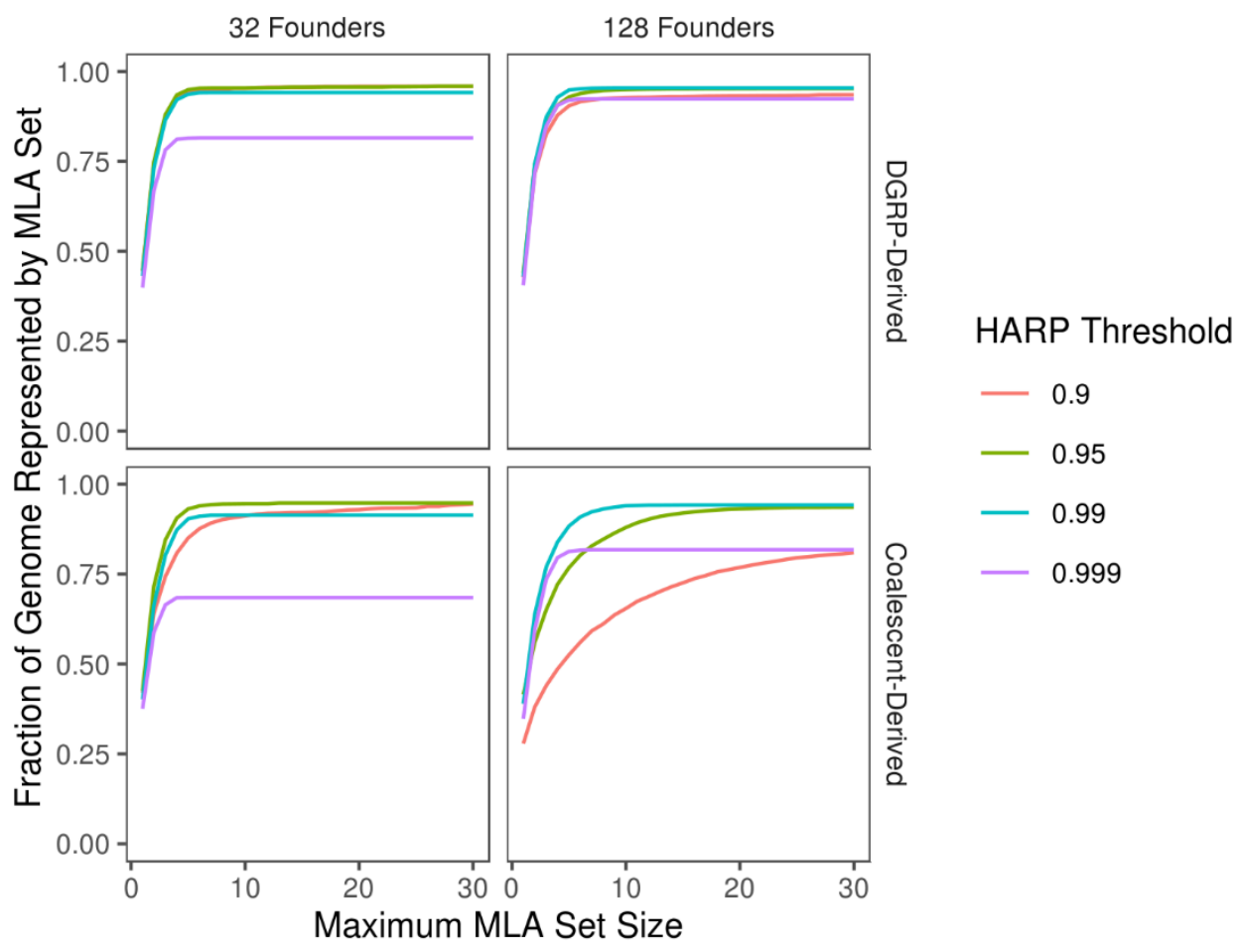

944 Supplemental Figure S7. Optimization curves for Most-Likely-Ancestor (MLA) selection.

946 To ensure reconstruction was both accurate and computationally feasible, we selected the smallest set of most-likely-ancestors that still represent the greatest fraction of to-

948 be-reconstructed genomes. Increasing the upper limit for the number of MLAs chosen improves the fraction of genome represented with diminishing returns. The HARP

950 threshold of 0.99 performed best for 128 -founder populations, for both DGRP-derived and coalescent-derived simulations. Conversely, 32-founder populations performed best

952 with a HARP threshold of 0.95 . Data shown reports means across 400 replicates made up of 100 simulated individuals (4 autosomes each for coalescent simulations, 4

954 autosome arms each for DGRP simulations) per parameter combination. Coalescentderived populations described here wer1e simulated with $\mathrm{N}_{\mathrm{e}}=1 \times 10^{6}$ and mutation rate $956 \mu=5 \times 10^{-9}$. 
bioRxiv preprint doi: https://doi.org/10.1101/671925; this version posted January 27,2021 . The copyright holder for this preprint (which was not certified by peer review) is the author/funder, who has granted bioRxiv a license to display the preprint in perpetuity. It is made available under aCC-BY-NC-ND 4.0 International license.

A

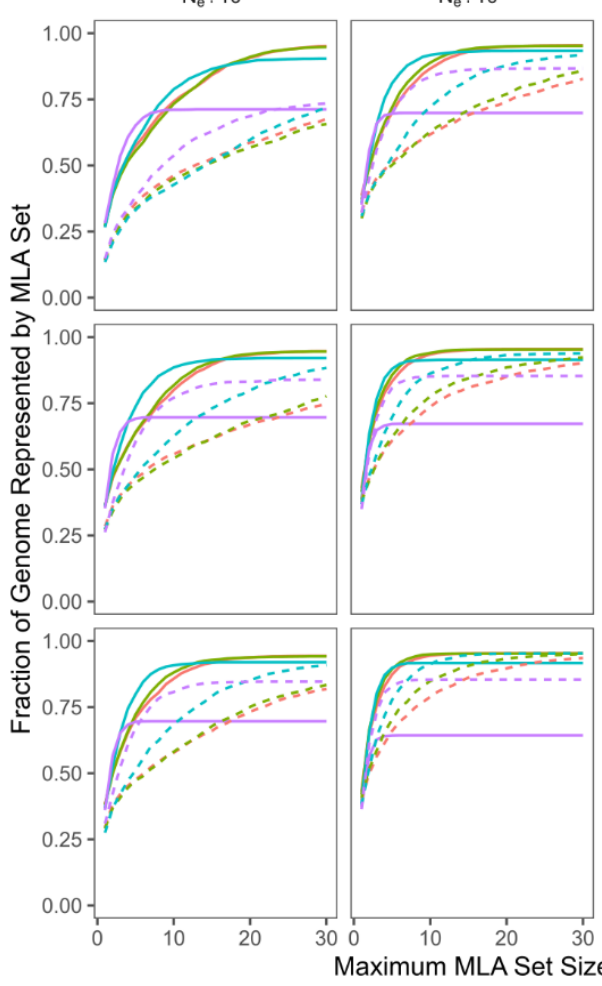

$N_{e}: 10^{6}$

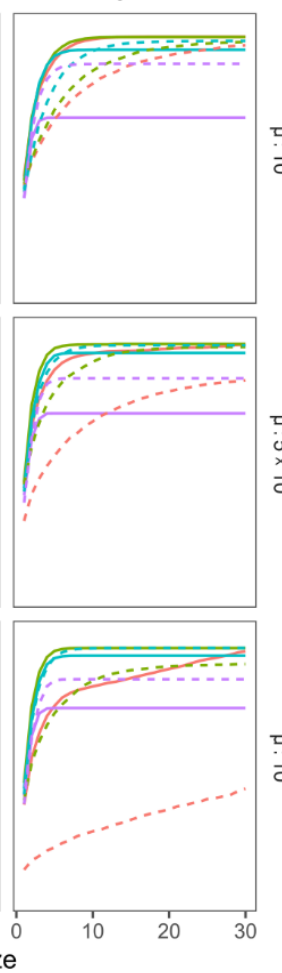

B

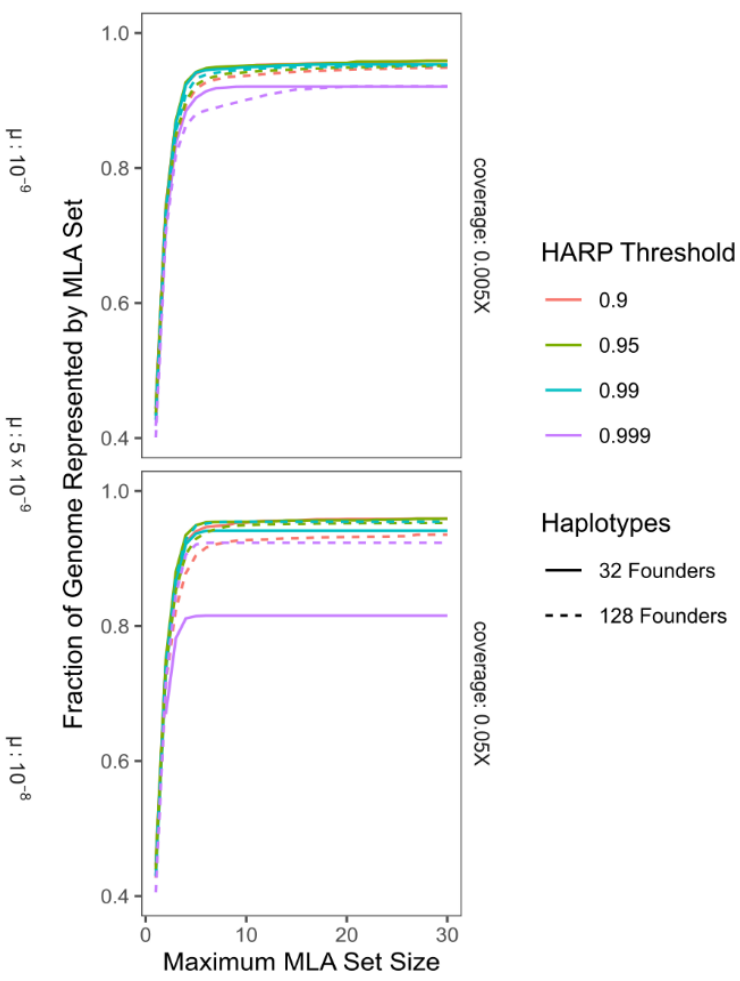

Supplemental Figure S8. Optimization curves for Most-Likely-Ancestor inclusion for 960 additional parameter combinations. A: SCRM (coalescent) derived populations across different parameters; $\mathrm{B}$ : accuracy is similar for both $0.05 \mathrm{X}$ and $0.005 \mathrm{X}$ sequencing 962 coverage in populations simulated with DGRP genomes. 


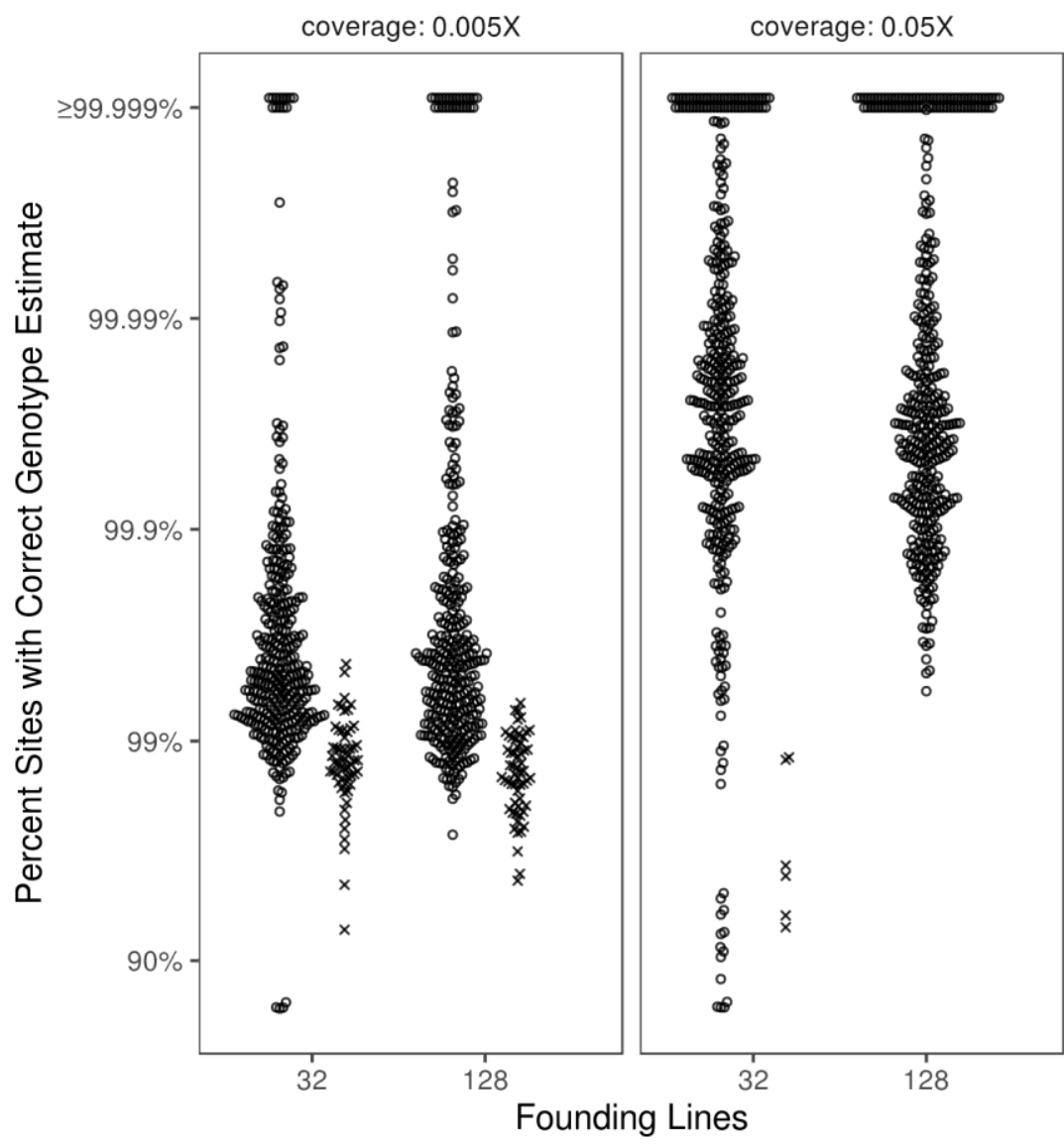

Estimated Recombinations

$0<10$

$X \geq 10$

Supplemental Figure S9. Accuracy of genome reconstruction for simulated, DGRP-

966 derived $F_{5}$ hybrid swarm individuals. Reconstructions were performed for populations simulated as being founded by either 32 or 128 inbred lines for two levels of ultra-low 968 sequencing coverage. Accuracy is represented on a logit scale, as most points occur above $90 \%$. Reconstructed chromosomes estimated to have $\geq 10$ recombination events

970 are denoted by an $\times$, offset from the bulk of the distribution. Each parameter combination includes 400 reconstructed chromosomes (from 100 simulated individuals). 


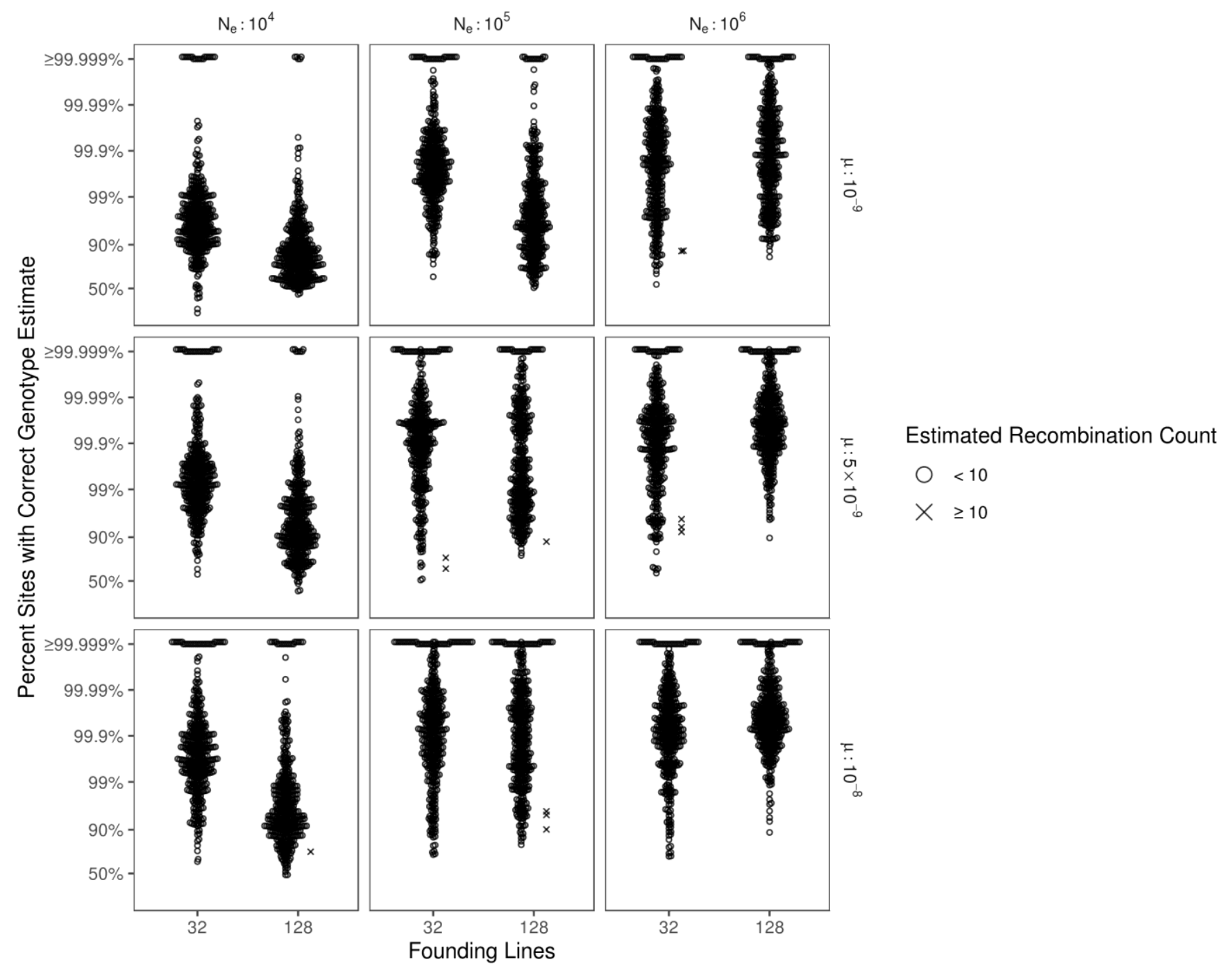

974 Supplemental Figure S10. Accuracy of genome reconstruction for simulated, coalescent-derived $\mathrm{F}_{5}$ hybrid swarm individuals. Reconstructions were performed for

976 populations simulated as being founded by either 32 or 128 inbred lines for various effective population sizes $\left(\mathrm{N}_{e}\right)$ and mutation rates $(\mu)$. Accuracy is represented on a logit

978 scale, as most points occur above $90 \%$. Reconstructed chromosomes estimated to have $\geq 10$ recombination events are denoted by an $x$, offset from the bulk of the

980 distributions. Each parameter combination includes 400 reconstructed chromosomes (from 100 simulated individuals). 


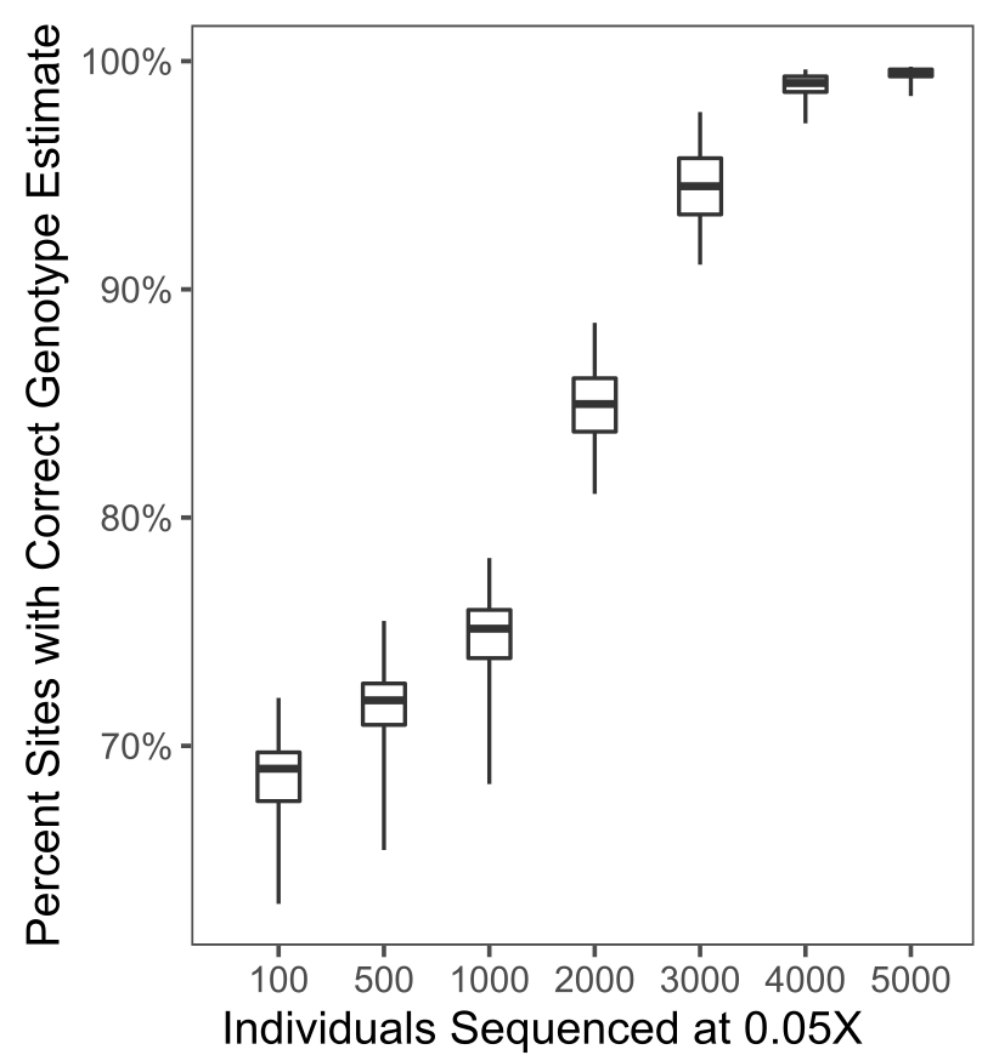

984 Supplemental Figure S11. Genotyping accuracy of STITCH for simulated 32-founder F5 hybrid swarm populations sequenced at $0.05 X$ coverage. Genotype accuracy

986 improves with greater numbers of sequenced individuals, as STITCH infers missing genotypes from other haplotypes in the population. Boxes represent the median and 988 interquartile range; whiskers extending to the lower and upper bounds of the $95 \%$ quantiles. See methods for parameters used. 


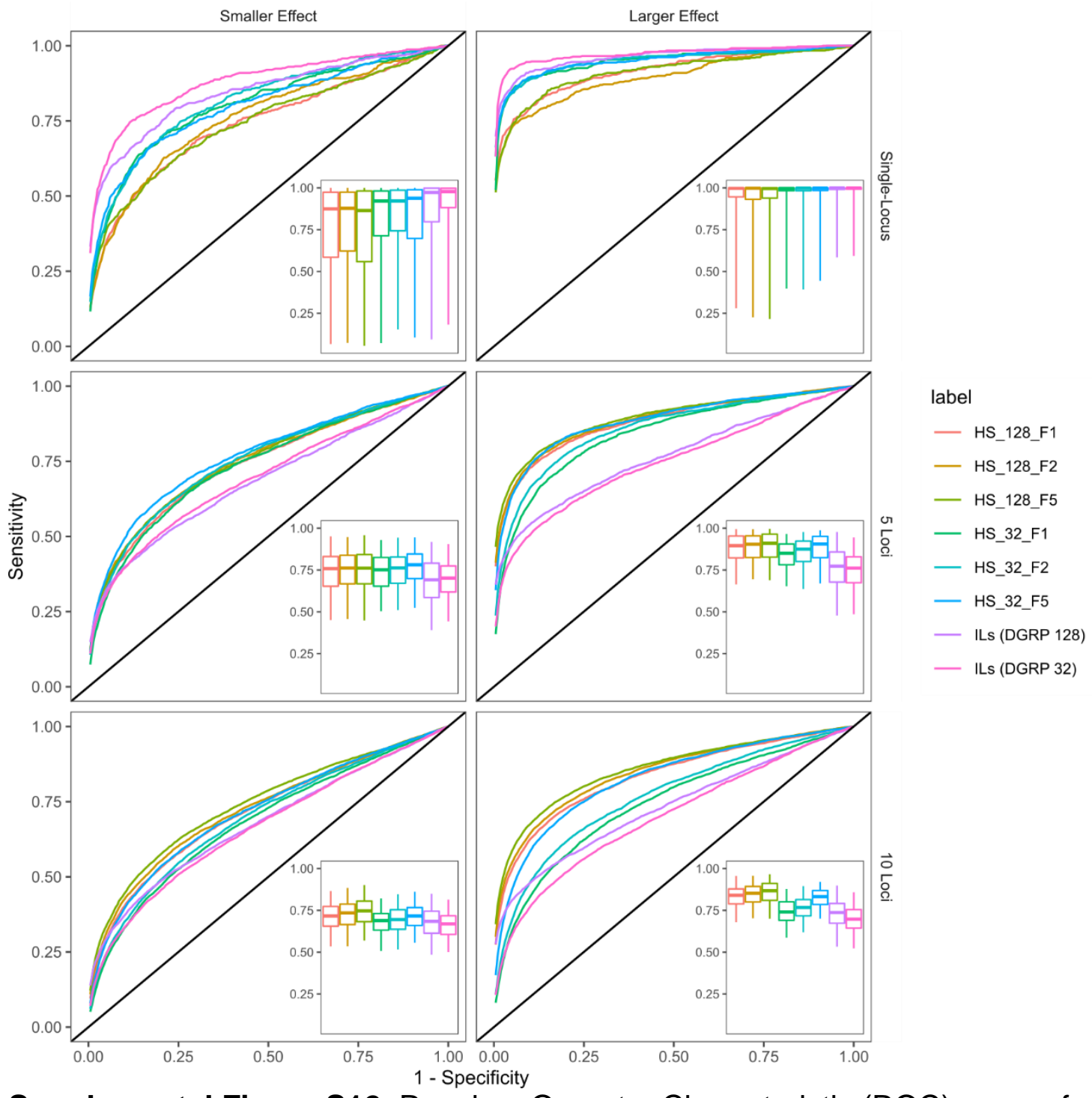

Supplemental Figure S12. Receiver Operator Characteristic (ROC) curves for

992 additional GWAS simulations. To generate single representative ROC curves across 500 GWAS simulations per population, we stepped through all specificity values and

994 calculated the mean sensitivity. Inset boxplots display the median and interquartile range of Area Under the Curve (AUC) distributions, with whiskers spanning the middle $99695 \%$ of data. ILs: inbred lines. HS: Hybrid Swarm populations founded by 32 or 128 lines. 

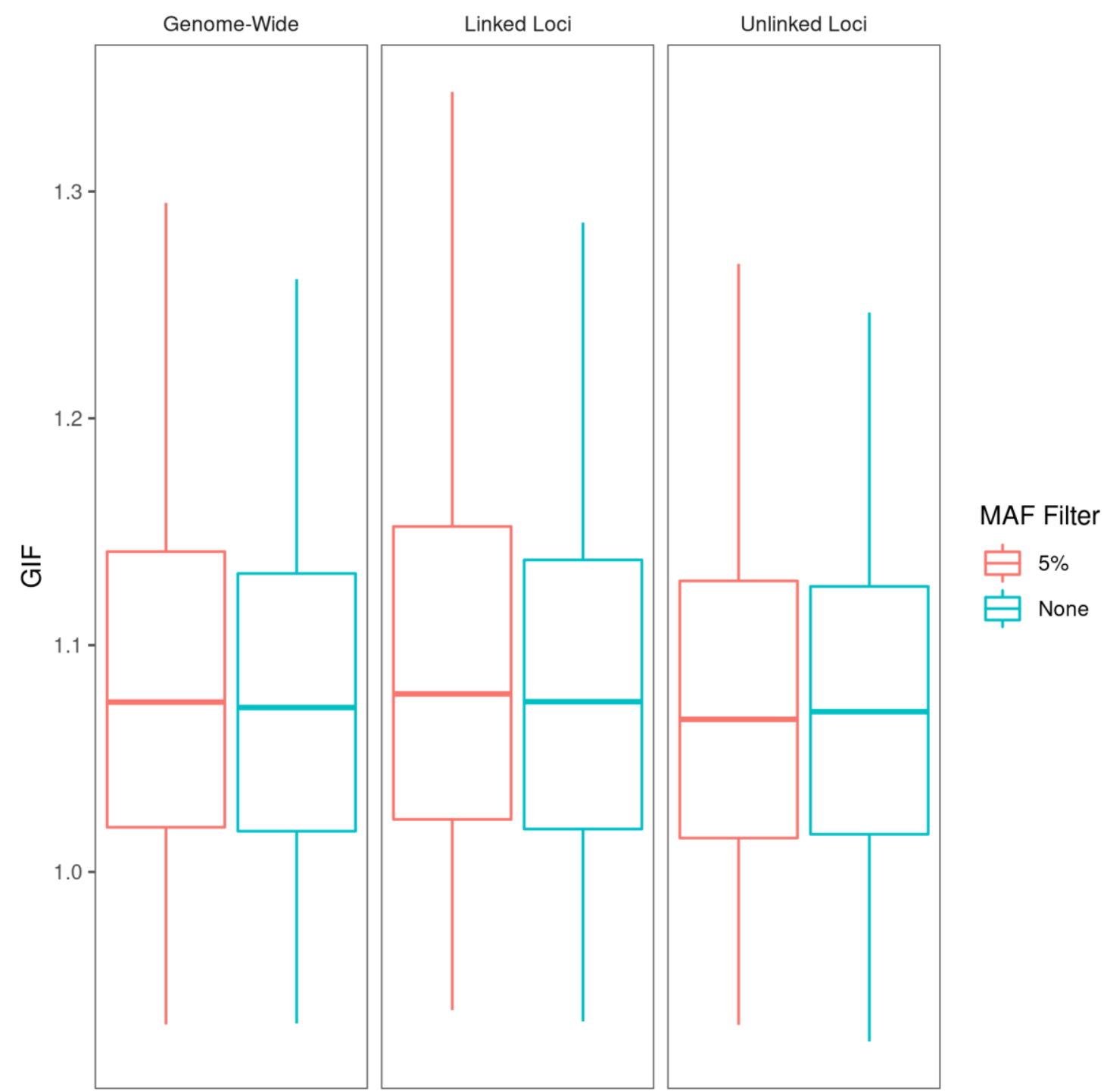

1002 Supplemental Figure S13. Genomic Inflation Factor with and without low-frequency filtering. Excluding alleles with a minor allele frequency below 5\% does not reduce

1004 genomic inflation factor in inbred lines. Box depicts the middle $50 \%$ quantiles with whiskers extending to the middle $95 \%$ of data points. Traits were modeled as a single 1006 locus of large effect. 


$\begin{array}{cccccccc}\text { Population } & \text { Coverage } & \text { N Founders } & \mathbf{N e} & \boldsymbol{\mu} & \boldsymbol{\rho} & \text { Mean } \boldsymbol{\Delta} & \boldsymbol{\sigma}_{\boldsymbol{\Delta}} \\ \text { DGRP } & 0.005 \mathrm{X} & 128 & - & - & 0.201 & 3.06 & 3.11 \\ \text { DGRP } & 0.005 \mathrm{X} & 32 & - & - & 0.201 & 3.15 & 2.81 \\ \text { DGRP } & 0.05 \mathrm{X} & 128 & - & - & 0.986 & -0.015 & 0.25 \\ \text { DGRP } & 0.05 \mathrm{X} & 32 & - & - & 0.502 & 0.17 & 2.15 \\ \text { Coalescent } & 0.05 \mathrm{X} & 128 & 10^{4} & 1 \times 10^{-9} & 0.029 & -2.72 & 1.60 \\ \text { Coalescent } & 0.05 \mathrm{X} & 32 & 10^{4} & 1 \times 10^{-9} & 0.219 & -1.84 & 1.50 \\ \text { Coalescent } & 0.05 \mathrm{X} & 128 & 10^{5} & 1 \times 10^{-9} & 0.288 & -1.57 & 1.64 \\ \text { Coalescent } & 0.05 \mathrm{X} & 32 & 10^{5} & 1 \times 10^{-9} & 0.761 & -0.53 & 0.99 \\ \text { Coalescent } & 0.05 \mathrm{X} & 128 & 10^{6} & 1 \times 10^{-9} & 0.742 & -0.46 & 1.09 \\ \text { Coalescent } & 0.05 \mathrm{X} & 32 & 10^{6} & 1 \times 10^{-9} & 0.754 & -0.42 & 1.22 \\ \text { Coalescent } & 0.05 \mathrm{X} & 128 & 10^{4} & 5 \times 10^{-9} & 0.203 & -2.03 & 1.73 \\ \text { Coalescent } & 0.05 \mathrm{X} & 32 & 10^{4} & 5 \times 10^{-9} & 0.622 & -0.89 & 1.17 \\ \text { Coalescent } & 0.05 \mathrm{X} & 128 & 10^{5} & 5 \times 10^{-9} & 0.652 & -0.64 & 1.41 \\ \text { Coalescent } & 0.05 \mathrm{X} & 32 & 10^{5} & 5 \times 10^{-9} & 0.782 & -0.26 & 1.16 \\ \text { Coalescent } & 0.05 \mathrm{X} & 128 & 10^{6} & 5 \times 10^{-9} & 0.956 & -0.17 & 0.44 \\ \text { Coalescent } & 0.05 \mathrm{X} & 32 & 10^{6} & 5 \times 10^{-9} & 0.759 & -0.31 & 1.26 \\ \text { Coalescent } & 0.05 \mathrm{X} & 128 & 10^{4} & 1 \times 10^{-8} & 0.238 & -1.65 & 1.86 \\ \text { Coalescent } & 0.05 \mathrm{X} & 32 & 10^{4} & 1 \times 10^{-8} & 0.745 & -0.66 & 1.05 \\ \text { Coalescent } & 0.05 \mathrm{X} & 128 & 10^{5} & 1 \times 10^{-8} & 0.776 & -0.34 & 1.12 \\ \text { Coalescent } & 0.05 \mathrm{X} & 32 & 10^{5} & 1 \times 10^{-8} & 0.846 & -0.25 & 0.93 \\ \text { Coalescent } & 0.05 \mathrm{X} & 128 & 10^{6} & 1 \times 10^{-8} & 0.937 & -0.22 & 0.56 \\ \text { Coalescent } & 0.05 \mathrm{X} & 32 & 10^{6} & 1 \times 10^{-8} & 0.833 & -0.32 & 0.95\end{array}$

Supplemental Table S1. Accuracy of estimated number of recombination events 1010 following chromosome reconstruction. A high concordance correlation coefficient (Lin's $\rho$ ) indicates agreement between estimated and true recombination counts for 400

1012 reconstructed chromosomes (coalescent-derived populations) or chromosome arms (DGRP-derived populations). Coalescent-derived populations are described across a

1014 range of values for effective population size $\mathrm{Ne}_{\mathrm{e}}$ and mutation rate $\mu$. $\Delta$ represents the difference between estimated and true recombination counts, and $\sigma_{\Delta}$ represents the

1016 mean of 400 standard deviations of $\Delta$. Reconstructions were performed with a maximum of 16 most-likely-ancestors with a HARP threshold of 0.99 (see methods for

1018 more details). 
bioRxiv preprint doi: https://doi.org/10.1101/671925; this version posted January 27, 2021. The copyright holder for this preprint (which was not certified by peer review) is the author/funder, who has granted bioRxiv a license to display the preprint in perpetuity. It is made available under aCC-BY-NC-ND 4.0 International license.

1020 Supplemental Table S2. Test statistics and p-values for 270 pairwise comparisons of simulated GWAS AUC distributions. Our $p$-value significance threshold at $\alpha=0.05$ is

$10221.85 \times 10^{-4}$. 


\section{Generating simulated reference panels across a range of diversity levels}

To evaluate low-coverage reconstruction for various degrees of genetic diversity, we

1028 generated reference panels using haplotypes produced by coalescent models across a range of genetic diversity levels. Haplotypes were generated using the $\mathrm{R}$ (R Core Team

1030 2016) package scrm (Staab et al. 2015) and subsequently restructured into VCF file format (Danecek et al. 2011). We generated ten independent panels for each of all 18

1032 combinations of population size $(\mathrm{Ne}=104,105,106)$, mutation rate $(\mu=10-9,5 \times 10-9,10$ 8 ), and number of haplotypes $(32,128)$. The value $\theta$ for each simulation was defined as

$10344 \mathrm{Ne \mu}$. We simulated a chromosome-length locus of $25 \mathrm{Mb}$ with a recombination rate of $1.5 \mathrm{cM} / \mathrm{Mb}$. SNP positions output by scrm (a decimal within the range of 0 to 1 ) were

1036 converted to base pair positions by multiplying the decimal by chromosome length (25 $\times 106$ base pairs for our simulations) and rounding down to the nearest integer. Any

1038 sites with more than two alleles were converted to a biallelic site by discarding tertiary or quaternary alleles. Genotype values were re-coded as polarized signed integers: +1 for

1040 reference and -1 for alternate alleles. For every position, reference and alternate alleles were defined by randomly selecting one of the twelve non-repeating pairs of

1042 nucleotides. Reference genome FASTA files were created with a custom python script that generated a 25 million length string of nucleotide characters with weighted

1044 probability to achieve $45 \%$ GC-content, followed by replacing variable positions with their respective reference alleles.

\section{Extended details for simulating GWAS}

1048 Simulated haplotypes. Although the forward simulator we developed is efficient, it would not have been computationally feasible to simulate 500 fully independent mapping

1050 populations (per parameter combination) in a reasonable amount of time. Instead, we generated ten independent forward-simulated populations, and for each of those,

1052 generated fifty randomly permuted subsets. For a single simulated mapping population, we began by sampling (with replacement) a random subset of 5,000 individuals, out of

105410,000 total individuals generated by forward-simulation. Then, we performed a permutation of haplotype ancestry with a new, randomly-ordered (equally sized) subset

1056 of founders. The permutation of ancestry was one-to-one, e.g. all haplotype blocks that were previously derived from founder $\mathrm{X}$ would be translated to founder $\mathrm{Y}$, and blocks

1058 previously derived from $Y$ would in turn be mapped to founder $Z$.

1060 For all simulated GWAS, we began with a set of 129 DGRP haplotypes with the least missing data, e.g. high coverage and low levels of heterozygosity. This allowed us to

1062 perform leave-one-out subsampling for the 128-founder populations. In addition to Hybrid Swarm populations, which we ran through the simulated sequencing and

1064 mapping pipeline, we generated four additional types of mapping populations for comparing GWAS performance: Highly outbred (F50) populations; Inbred Lines (ILs) to

1066 represent the DGRP; and Recombinant Inbred Lines (RILs), similar to the Drosophila Synthetic Population Resource, or DSPR (King et al. 2012). 
The F50 populations were generated with 128 founders in same manner as the Hybrid 1070 Swarm, except that populations were simulated over fifty non-overlapping generations of recombination instead of five generations. The ten resulting forward-simulated

1072 populations were resampled and permuted as we did with the Hybrid Swarms.

1074 We simulated ten initial sets of 800 RILs using the same forward-simulator as previously described, each initialized with a random subset of eight DGRP haplotypes. Populations

1076 randomly recombined at a population size of 10,000 for fifty non-overlapping generations, after which 800 random male-female pairs of individuals were isogenzied

1078 through 25 generations of full-sibling mating. This scenario roughly corresponds to the DSPR. For computational simplicity, after the 25 generations of isogenization we

1080 removed any remaining residual heterozygosity by forcing the identity of a second chromosome copy to be identical to the first copy. We then sampled 5,000 draws (with

1082 replacement) of the 800 RILs followed by ancestry permutation as described above.

1084 To simulate GWAS on Inbred Lines, no forward-simulation was necessary. For a single simulated population, we first randomly selected 128 DGRP lines, then randomly

1086 sample with replacement 5,000 times. As with hybrid swarm and RILs, for any parameter combination we generated a total of 500 mapping populations.

Liability model. Using the liability framework, we assign case or control phenotypes to mapping populations derived from DGRP chromosome $2 \mathrm{~L}$ haplotypes. First, a genotype-dependent risk score is calculated for every individual, which is translated into 1092 probabilities for case or control group assignment. We parameterized our model such that individuals begin with equivalent odds of being assigned to case or control groups,

1094 and that probability is modified depending on allele status at causal loci.

1096 For a given simulation, $\mathrm{N}$ causal loci are randomly selected with equal probability out of all segregating sites in the mapping population. The effect of individual causal loci are

1098 simulated as a Gaussian variable drawn with a small extent of noise $(\mathrm{sd}=0.005)$ with an expected (mean) value dependent on the allele frequency at that locus. Intermediate

1100 frequency loci (i.e. allele frequency $=0.5$ ) are simulated to contribute on average no effect on case/control assignment; alleles near fixation decrease risk; rare alleles

1102 increase risk. The linear center of the curve, where most individual risk scores exist under the parameter combinations used in our simulations, approximates additive

1104 genetic architecture. The sigmoid tails allow for the continuous risk score to translate to a phenotype necessarily bounded by zero and one. The relationship between allele

1106 frequency, risk score, and phenotypic assignment is diagrammed in Supplemental Figure S9. Within the linear portion of the curve (which is the domain for the majority of

1108 simulations), a singleton minor allele will modify 'case' assignment probability by approximately $+5 \%$ (small effect) or $+10 \%$ (large effect), beginning at an initial

1110 assignment probability of $50 \%$. The major allele, conversely, would decrease assignment by an opposite amount. An allele at $50 \%$ frequency will not modify

1112 case/control assignment (aside from a very small degree of random noise equal to about $+/-0.06 \%$ ). Note that we performed GWAS simulations assuming $100 \%$ genotype

1114 accuracy. 
1116 We implemented a method of efficiently aggregating allele counts compatible with our haplotype map format. Briefly, haplotype map breakpoints across all individuals are

1118 sorted in ascending order. When iterating through ascending unique start and stop positions, between any pair of breakpoints, all SNPs will be comprised of the same

1120 number of each founding haplotype. Haplotype IDs could then be counted and sorted in the same column position order as the table containing polarized allele status $(-1$ for

1122 alternate, +1 for reference). Multiplying the genotype table by the haplotype count vector results in final allele counts, polarized negative for alternate alleles and positive for

1124 reference alleles. 